\title{
The Molecular Interstellar Medium in Ultraluminous Infrared Galaxies
}

\author{
P. M. Solomon \\ Astronomy Program, State University of New York, Stony Brook, NY 11794 \\ D. Downes \\ Institut de Radio Astronomie Millimétrique, 38406 St. Martin d'Hères, France \\ S. J. E. Radford \\ National Radio Astronomy Observatory, Tucson AZ 85721 \\ and \\ J. W. Barrett \\ Astronomy Program, State University of New York, Stony Brook, NY 11794
}

Received March 28, 1996; accepted __ 


\begin{abstract}
We present observations with the IRAM $30 \mathrm{~m}$ telescope of CO in a large sample of ultraluminous IR galaxies out to redshift $z=0.3$. Most of the ultraluminous galaxies in this sample are interacting, but not completed mergers. The $\mathrm{CO}(1-0)$ luminosity of all but one of the ultraluminous galaxies is high, with values of $\log \left(L_{\mathrm{CO}}^{\prime} / \mathrm{K} \mathrm{km} \mathrm{s}^{-1} \mathrm{pc}^{2}\right)=9.92 \pm 0.12$. The extremely small dispersion of only $30 \%$ is less than that of the far infrared luminosity. The integrated CO line intensity is strongly correlated with the $100 \mu \mathrm{m}$ flux density, as expected for a black body model in which the mid and far IR radiation is optically thick. We use this model to derive sizes of the FIR and CO emitting regions and the enclosed dynamical masses. Both the IR and $\mathrm{CO}$ emission originate in regions a few hundred parsecs in radius. The median value of $L_{\mathrm{FIR}} / L_{\mathrm{CO}}^{\prime}=160 \mathrm{~L}_{\odot} / \mathrm{K} \mathrm{km} \mathrm{s}^{-1} \mathrm{pc}^{2}$, within a factor of two or three of the black body limit for the observed far IR temperatures. The entire ISM is a scaled up version of a normal galactic disk with the ambient densities a factor of 100 higher, making even the intercloud medium a molecular region. We compare three different techniques of $\mathrm{H}_{2}$ mass estimation and conclude that the ratio of gas mass to CO luminosity is about a factor of four times lower than for Galactic molecular clouds, but that the gas mass is a large fraction of the dynamical mass. Our analysis of $\mathrm{CO}$ emission from ultraluminous galaxies reduces the $\mathrm{H}_{2}$ mass from previous estimates of $2-5 \times 10^{10} \mathrm{M}_{\odot}$ to $0.4-1.5 \times 10^{10} \mathrm{M}_{\odot}$, which is in the range found for molecular gas rich spiral galaxies. A collision involving a molecular gas rich spiral could lead to an ultraluminous galaxy powered by central starbursts triggered by the compression of infalling preexisting GMC's.

The extremely dense molecular gas in the center of an ultraluminous galaxy is an ideal stellar nursery for a huge starburst.
\end{abstract}

Subject headings: galaxies: nuclei — galaxies: interstellar matter — galaxies: starburst - galaxies: ISM: dust, extinction — radio lines: galaxies — infrared: galaxies 


\section{INTRODUCTION: A NEW CO STUDY OF ULTRALUMINOUS GALAXIES}

We report here an observational study of the molecular content of a large sample of extremely luminous infrared galaxies. Ultraluminous galaxies, those with IR luminosities' $>10^{12} \mathrm{~L}_{\odot}$ (cf. Wright, Joseph and Meikle, 1984; Sanders et al. 1988a), are the most luminous objects in the local universe. They typically radiate $90 \%$ or more of their energy in the far infrared. Most of these objects were discovered by the IRAS survey and many were previously uncatalogued. All ten of the brightest (nearest) ultraluminous galaxies (Sanders et al. 1988a) are either merging systems or have tidal tails indicating a recent merger. Besides the very large IR luminosity, the molecular interstellar medium in such galaxies differs from that in normal spiral galaxies in several fundamental respects. First, even though these galaxies have high CO luminosities and molecular masses, the ratio of far infrared to CO luminosity is about an order of magnitude greater than for normal spiral galaxies (e.g., Sanders et al. 1986; Solomon \& Sage 1988). In star formation models, this implies a much higher star formation rate per Solar mass of molecular gas than in normal galaxies, even gas rich spirals. Second, a large fraction of their molecular gas is at densities much higher than in ordinary giant molecular clouds, as shown by HCN observations which trace gas at densities $>10^{5} \mathrm{~cm}^{-3}$ (Solomon, Downes, \& Radford 1992a). Third, direct interferometric measurements of nearby ultraluminous galaxies (e.g., Scoville et al. 1991; Radford et al. 1991b) show most of the CO, HCN, and molecular mass is concentrated in a small central region (less than $1 \mathrm{kpc}$ ). Indeed, estimates of the molecular mass in the central condensation often equal the dynamical mass (e.g., Scoville et al. 1991). This conflict between dynamical mass and $\mathrm{H}_{2}$ mass derived from $\mathrm{CO}$ luminosity leads to a new interpretation of the CO luminosity (Downes, Solomon, \& Radford 1993; hereafter DSR) for a medium which may fill a disk or sphere in the central few hundred parsecs of a galaxy.

In this paper we present the results of a systematic $\mathrm{CO}(1-0)$ survey with the IRAM $30 \mathrm{~m}$ telescope of a large sample of ultraluminous galaxies out to $z=0.27$. This is part of a multiline study with a goal of understanding the difference between the star-forming environment in ultraluminous galaxies and large spiral galaxies. The observational data are in section 2. We derive $\mathrm{CO}$ and IR luminosities and temperatures in section 3 and compare them with the values for normal spirals. We have previously suggested that for the extreme conditions of ultraluminous galaxies, the ratio of IR to CO luminosity approaches the ratio expected for a black body (DSR) This implies the dust is optically thick even at far IR wavelengths (see also Condon et al. 1991) and the ratio is not a simple indicator of luminosity to molecular mass. In section 4 , we review the black body model and discuss

\footnotetext{
${ }^{1}$ We use $H_{0}=75 \mathrm{~km} \mathrm{~s}^{-1} \mathrm{Mpc}^{-1}$ and $q_{0}=0.5$ throughout this paper.
} 
the validity of our black-body approximation for the central regions. In section 5, we derive the dynamical mass from the model and the observations, and compare it with the $\mathrm{H}_{2}$ mass derived from CO luminosity. We also derive a lower limit to the molecular mass by assuming the $\mathrm{CO}$ is optically thin, and we compare the mass calculated by different approximations. We discuss the limits of the model, the extent to which gas may be a dominant part of the dynamical mass in the centers of these galaxies and the implications for the nature of the luminosity source.

\section{OBSERVATIONS}

\subsection{The Sample and Observing Methods}

\subsubsection{Source Selection}

The sample contains 37 infrared luminous galaxies in the redshift range $\mathrm{z}=0.03$ to 0.27. Of these 11, including the well known sources Arp 220 and Mrk 231, have $60 \mu \mathrm{m}$ fluxes $S_{60}>5.0 \mathrm{Jy}$ and are part of the nearby bright galaxy sample (Sanders et al. 1988a; Sanders, Scoville, \& Soifer 1991). They have previously been observed in CO by several groups. Twenty galaxies were chosen from a redshift survey (Strauss et al. 1992) of all IRAS sources with $60 \mu \mathrm{m}$ fluxes $S_{60}>1.9 \mathrm{Jy}$. We selected primarily the most far infrared luminous sources (see equation 4 ) at $\delta>-10^{\circ}$; a few lower luminosity sources were also included. We excluded a few sources from the redshift survey whose unrealistically high $100 / 60 \mu \mathrm{m}$ flux ratios indicate they are probably contaminated by Galactic emission. In order to include more galaxies at very high infrared luminosity we also observed 7 sources with $60 \mu \mathrm{m}$ fluxes between 1.2 and 1.9 Jy from the extension of the redshift survey. ( Fisher et al., 1995) These are the most distant, $0.15 \leq z \leq 0.27$, galaxies in the sample.

Although not complete, our sample includes a large fraction of the most luminous IRAS galaxies in the northern sky. Of the ten most luminous galaxies in the redshift survey above $\delta>-10^{\circ}$, our sample includes eight. One of the missing sources is the radio-loud quasar $3 \mathrm{C} 273$. Twenty galaxies in our sample are more luminous than the prototype IR galaxy Arp 220 and 25 have a far infrared luminosity, $L_{\mathrm{FIR}}>1 \times 10^{12} \mathrm{~L}_{\odot}$, obtained from the color corrected 60 and $100 \mu \mathrm{m}$ flux (see equation 4). The most luminous object in the sample, $14070+0525$, with $4 \times 10^{12} \mathrm{~L}_{\odot}$ in the far IR, is also the most distant.

In our sample, the mean far IR color $S_{60} / S_{100}=0.81$, is slightly cooler than in the

smaller sample studied by Sanders et al. (1988a), where the mean $S_{60} / S_{100}=0.93$ (Sanders, private communication). Only $19 \%$ of the galaxies in our sample have $S_{60} / S_{100}>1.0$, 
while $50 \%$ have $S_{60} / S_{100}>1.0$ in Sanders et al.'s sample. A possible explanation is that although most of our sample was taken from a redshift survey of $60 \mu \mathrm{m}$ sources, we selected candidates based on total far infrared luminosity including the contribution from $100 \mu \mathrm{m}$. The sample covers higher redshifts than the earlier studies (Sanders et al. 1988a; 1991). Figure 1 shows the redshift distribution for the galaxies described here, which extends to $c z=80000 \mathrm{~km} \mathrm{~s}^{-1}$. For comparison, Sanders, et al. (1991) included three ultraluminous galaxies in their highest range of redshift, $c z=15000-25000 \mathrm{~km} \mathrm{~s}^{-1}$. In the redshift range

$c z=15000-80000 \mathrm{~km} \mathrm{~s}^{-1}$, our sample has 25 galaxies with $60 \mu \mathrm{m}$ fluxes between 1.0 and 5 Jy. Of these, 22 galaxies have a FIR luminosity $L_{\mathrm{FIR}}>1 \times 10^{12} \mathrm{~L}_{\odot}$.

\subsubsection{Positions of the IRAS Galaxies}

With the IRAM $30 \mathrm{~m}$ telescope, which has a beamwidth of $22^{\prime \prime}$ at $115 \mathrm{GHz}$ and $13^{\prime \prime}$ at $230 \mathrm{GHz}, \mathrm{CO}$ observations require source positions accurate to a few arcsec. This is more accurate than positions in the IRAS catalogs (Moshir et al. 1992; Beichman et al. 1988), which typically have error ellipses with major axes of $15^{\prime \prime}-20^{\prime \prime}$. For the faintest sources, the IRAS positions may be no better than $30^{\prime \prime}$. Hence we determined positions of candidate galaxies near the center of the IRAS error ellipse for each source from the Palomar Sky Survey prints with a measuring engine. The weakest sources have visual magnitudes near the Sky Survey limit (mag 17 to 18). We could usually identify the most likely galaxy by inspection and we detected $\mathrm{CO}$ in all of these with no exceptions. Table $\mathbf{1}$ gives the positions where we detected CO. We estimate the accuracy to be $\pm 3^{\prime \prime}$ for sources with positions from the Sky Survey. For a few of the stronger sources we used VLA continuum positions (Condon et al. 1991).

Some potential sources selected from the extension to the redshift survey with $60 \mu \mathrm{m}$ fluxes between 1.2 and $1.9 \mathrm{Jy}$ are in confused fields where we found several very faint candidates. In 5 cases we stopped looking after one or two tries due to limited observing time. The limits in these cases were not low enough to be interesting given the very weak far IR flux. In other words we simply did not invest the observing time required to reach the expected integrated intensity given the low $100 \mu \mathrm{m}$ flux (see Fig. 4, section 4), and the confused position. These sources are not included in the sample.

\subsubsection{CO Observations}

The CO observations were made with the IRAM $30 \mathrm{~m}$ telescope on Pico Veleta near Granada, Spain. All observations were done with a wobbling secondary with a throw of $120^{\prime \prime}$ 
- 240" in a double switching mode, alternating on-off and off-on. The SIS receivers had typical SSB noise temperatures of $130-230 \mathrm{~K}$. The $512 \times 1 \mathrm{MHz}$ filter banks covered 1400 $-1600 \mathrm{~km} \mathrm{~s}^{-1}$ at the redshifted $\mathrm{CO}(1-0)$ line and the data were smoothed to resolutions of $8 \mathrm{MHz}\left(\approx 24 \mathrm{~km} \mathrm{~s}^{-1}\right)$ or $16 \mathrm{MHz}\left(\approx 48 \mathrm{~km} \mathrm{~s}^{-1}\right)$ for analysis. To test the reality of the weakest lines, we used two local oscillator settings, shifting the lines by $200-300 \mathrm{~km} \mathrm{~s}^{-1}$. The data were calibrated with cold and ambient loads and pointing was checked on planets and quasars. Atmospheric opacities were typically $<0.1$ at the observing frequencies of $91-$ $110 \mathrm{GHz}$. At these frequencies, the telescope's forward efficiency is $90 \%$ and its main beam efficiency is $60 \%$. In this paper, spectra and intensities, $I_{\mathrm{CO}}$, are in main beam brightness temperature, $T_{\mathrm{mb}}$, which is appropriate for small sources. For the $30 \mathrm{~m}$ telescope, $T_{\mathrm{mb}}=1 \mathrm{~K}$ corresponds to a flux density of $4.5 \mathrm{Jy}$ from a point source in the $3 \mathrm{~mm}$ band.

Spectra of the sources are shown in Fig. 2 and integrated intensities are listed in Table 1. A few of these spectra have been published previously (Radford, Solomon \& Downes 1991a; Solomon, Downes \& Radford 1992a). The peak intensities range from $T_{\mathrm{mb}}=2 \mathrm{mK}$ for the most distant object at $z=0.265$ to $\sim 100 \mathrm{mK}$ for the nearest sources. The CO redshifts agree well with the optical redshifts; the difference between the two is typically $\leq 30 \mathrm{~km} \mathrm{~s}^{-1}$. The linewidths in Table $\mathbf{1}$ are either the full width at half maximum of Gaussian fits or one-half the full width to zero intensity for line profiles that are strongly non-gaussian. Unlike spectra from normal spirals, many of these profiles are centrally peaked.

In addition to $\mathrm{CO}$ data, we present R-band CCD images of 12 of the galaxies between RA $10 \mathrm{~h}$ and $23 \mathrm{~h}$, taken at the University of Hawaii $2.2 \mathrm{~m}$ telescope. The galaxy morphology shown by these images is discussed in Section 6; for easy reference, we place our CO spectra next to the images in order of decreasing redshift (Fig. 7).

\section{CO AND INFRARED LUMINOSITIES}

Table 2 lists the CO and far IR luminosities of the sample galaxies. Here we describe how we calculate luminosities from observed fluxes and we compare the luminosities with those of normal galaxies.

\subsection{CO Luminosities}

\subsubsection{Basic Expressions}


The CO line luminosity can be expressed several ways. Monochromatic luminosity $L\left(\nu_{\text {rest }}\right)$, observed flux density $S\left(\nu_{\text {obs }}\right)$, and luminosity distance $D_{L}$ are related by $\nu_{\text {rest }} L\left(\nu_{\text {rest }}\right)=4 \pi D_{L}^{2} \nu_{\text {obs }} S\left(\nu_{\text {obs }}\right)$, so

$$
L_{\mathrm{CO}}=1.04 \times 10^{-3} S_{\mathrm{CO}} \Delta V \nu_{\mathrm{rest}}(1+z)^{-1} D_{L}^{2} \quad,
$$

where $L_{\mathrm{CO}}$ is the $\mathrm{CO}$ line luminosity in $\mathrm{L}_{\odot}, S_{\mathrm{CO}} \Delta V$ is the velocity integrated flux in $\mathrm{Jy} \mathrm{km} \mathrm{s}{ }^{-1}, \nu_{\text {rest }}=\nu_{\mathrm{obs}}(1+z)$ is the rest frequency in $\mathrm{GHz}$, and $D_{L}$ is the luminosity distance in Mpc.

Often CO line luminosity is expressed as velocity integrated source brightness temperature, $T_{b} \Delta V$, times source area, $\Omega_{\mathrm{s}} D_{A}^{2}$, where $\Omega_{\mathrm{s}}$ is the solid angle subtended by the source and $D_{A}=D_{L} /(1+z)^{2}$ is the angular size distance. The observed integrated line intensity, $I_{\mathrm{CO}}=\int T_{\mathrm{mb}} d V$, is obtained from the beam-diluted brightness temperature. This must be corrected for redshift to get the intrinsic source brightness temperature, $T_{b} \Delta V \Omega_{\mathrm{s}}=I_{\mathrm{CO}} \Omega_{\mathrm{s} \star \mathrm{b}}(1+z)$, where $\Omega_{\mathrm{s} \star \mathrm{b}}$ is the solid angle of the source convolved with the telescope beam. Then the line luminosity $L_{\mathrm{CO}}^{\prime}=T_{b} \Delta V \Omega_{\mathrm{s}} D_{A}^{2}=\Omega_{\mathrm{s} \nless \mathrm{b}} D_{L}^{2} I_{\mathrm{CO}}(1+z)^{-3}$, or

$$
L_{\mathrm{CO}}^{\prime}=23.5 \Omega_{\mathrm{s} \star \mathrm{b}} D_{L}^{2} I_{\mathrm{CO}}(1+z)^{-3}
$$

when $L_{\mathrm{CO}}^{\prime}$ is in $\mathrm{Kkm} \mathrm{s}^{-1} \mathrm{pc}^{2}, \Omega_{\mathrm{s} \times \mathrm{b}}$ is in $\operatorname{arcsec}^{2}, D_{L}$ is in $\mathrm{Mpc}$, and $I_{\mathrm{CO}}$ is in $\mathrm{Kkm} \mathrm{s}^{-1}$. If the source is much smaller than the beam, $\Omega_{\mathrm{s} \star \mathrm{b}} \approx \Omega_{\mathrm{b}}$. Here we see that for a fixed beam size and source luminosity, the integrated line intensity does not scale as $D_{L}^{-2}$, but rather as $(1+z)^{3} D_{L}^{-2}$ (Solomon, Radford, \& Downes 1992). The line luminosity, $L_{\mathrm{CO}}^{\prime}$, can also be expressed for a source of any size in terms of the total line flux, $L_{\mathrm{CO}}^{\prime}=\left(c^{2} / 2 k\right) S_{\mathrm{CO}} \Delta V \nu_{\mathrm{obs}}^{-2} D_{L}^{2}(1+z)^{-3}$, or

$$
L_{\mathrm{CO}}^{\prime}=3.25 \times 10^{7} S_{\mathrm{CO}} \Delta V \nu_{\mathrm{obs}}^{-2} D_{L}^{2}(1+z)^{-3} .
$$

with $S_{\mathrm{CO}} \Delta V$ in $\mathrm{Jy} \mathrm{km} \mathrm{s}{ }^{-1}, \nu_{\mathrm{obs}}$ in $\mathrm{GHz}$, and $D_{L}$ in Mpc.

The quantity $L_{\mathrm{CO}}^{\prime}$ is useful because it is surface brightness times area, in brightness temperature units. Thus two lines with the same $T_{b}$ and extent will have the same $L_{\mathrm{CO}}^{\prime}$, regardless of transition or line frequency. Conversely, the $L_{\mathrm{CO}}^{\prime}$ ratio of two lines is the average over the source of the lines' intrinsic $T_{b}$ ratio, which is an indicator of physical conditions in the gas.

\subsubsection{CO Luminosities and Gas Masses for this Sample}

Table 2 lists the CO luminosities and the nominal gas masses $M^{\prime}\left(\mathrm{H}_{2}\right)$ computed with a standard Milky Way $\mathrm{H}_{2}$ mass-to-CO luminosity ratio of $4.6 \mathrm{M}_{\odot} / \mathrm{K} \mathrm{km} \mathrm{s}^{-1} \mathrm{pc}^{2}$ (Solomon 
et al. 1987). The average for the sample is $L_{\mathrm{CO}}^{\prime}=8 \times 10^{9} \mathrm{~K} \mathrm{~km} \mathrm{~s}^{-1} \mathrm{pc}^{2}$, or $4 \times 10^{10} \mathrm{M}_{\odot}$ of molecular gas. These are $\sim 20$ times greater than the CO luminosity and molecular mass of the Milky Way interior to the Solar circle (Solomon \& Rivolo 1989). Previous $\mathrm{CO}$ surveys of spiral galaxies show there are many isolated, non-interacting spirals with CO luminosities 2 - 5 times higher than the Milky Way. Examples include NGC 6946, NGC 7479, and NGC 1530 (cf. Solomon \& Sage 1988). A particularly gas rich, isolated galaxy with normal far IR properties is NGC 3147. Its CO luminosity and molecular mass are 15 times larger than the Milky Way's and close to the average for these ultraluminous IR galaxies. High molecular masses appears, however, to be more common in interacting galaxies than in normal galaxies. In a group of 29 interacting galaxies with separations $<5 D_{25}$ and some morphological disturbances, Solomon \& Sage (1988) found 15 had molecular masses greater than $5 \times 10^{9} \mathrm{M}_{\odot}$. Below (section 5) we derive the molecular mass of ultraluminous galaxies by several methods, including estimates of the dynamical mass and of the $\mathrm{H}_{2}$ mass for optically thin $\mathrm{CO}$ emission. We conclude the standard Milky Way $M\left(\mathrm{H}_{2}\right) / L_{\mathrm{CO}}^{\prime}$ ratio overestimates the $\mathrm{H}_{2}$ masses of these galaxies by a factor of three.

\subsection{Infrared Luminosities and Dust Temperatures}

¿From the fluxes in the IRAS Faint Source Catalog we calculated FIR luminosities (Table 2).

$$
L_{\mathrm{FIR}}=3.94 \times 10^{5}\left(2.58 S_{60}+S_{100}\right) r\left(S_{60} / S_{100}\right) D_{L}^{2},
$$

with $L_{\mathrm{FIR}}$ in $\mathrm{L}_{\odot}, S$ in Jy, and $D_{L}$ in Mpc. The color correction, $r$, is a function of the $60-100 \mu \mathrm{m}$ flux ratio and the assumed dust emissivity (Lonsdale et al. 1985). This is a multiplicative factor between 1.5 and 2.1 that allows the FIR luminosity to be derived from the 60 and $100 \mu \mathrm{m}$ fluxes.

Note, we use far IR luminosity, $L_{\mathrm{FIR}}$ rather than IR luminosity, $L_{\mathrm{IR}}$, which includes the $25 \mu \mathrm{m}$ flux (Sanders et al. 1991). For the faintest galaxies in the sample the $25 \mu \mathrm{m}$ flux has a large uncertainty and in any case contributes only about $20 \%$ to the total IR luminosity except for four warm objects with $25 / 60 \mu \mathrm{m}$ ratios $>0.2$ where $L_{\mathrm{IR}}$ is about $50 \%$ higher than $L_{\mathrm{FIR}}$. In terms of IR luminosity the number of sources in the sample with $L_{\mathrm{IR}}>1 \times 10^{12} \mathrm{~L}_{\odot}$ remains at 25 ; there are another 7 with $L_{\mathrm{IR}}>0.8 \times 10^{12} \mathrm{~L}_{\odot}$.

The dust temperature derived from the far IR colors depends on the assumed emissivity for optically thin dust but is independent of emissivity for an optically thick source. While the dust in normal galaxies, including the Milky Way, is transparent in the far IR, there is

strong evidence (next section and Condon et al. 1991) the dust in ultraluminous galaxies is opaque, even at 60 and $100 \mu \mathrm{m}$. We used, therefore, a black body emissivity index, $n=0$, 
to calculate dust temperatures and color corrections. This gives higher dust temperatures than the optically thin approximation. The observed dust temperatures were also multiplied by $(1+z)$ to obtain rest frame temperatures.

Figure 3 is a diagram of FIR luminosity vs. CO luminosity for the galaxies in our sample. For comparison, some well-known galaxies and the trend for normal and weakly interacting spirals (Solomon \& Sage 1988) are also shown. Ultraluminous galaxies have a systematically higher infrared to CO luminosity ratio. In the following section we develop a model which explains the high ratio and shows that there is a clear upper limit to $L_{\mathrm{FIR}} /$ $L_{\mathrm{CO}}$. The model also leads to a re-interpretation of the molecular mass determination for ultraluminous galaxies.

\section{BLACK BODY MODEL}

We argued previously (DSR) that for ultraluminous galaxies, the tight correlation of CO line intensity and $100 \mu \mathrm{m}$ flux strongly indicates the dust is optically thick at $100 \mu \mathrm{m}$. The essential steps in that argument were:

a) Small sizes of CO regions imply black bodies in the FIR. Interferometers show the molecular gas in ultraluminous galaxies is in small central regions. Examples are Arp 220, with a CO and HCN core radius of 320 pc (Scoville et al. 1991; Okumura et al. 1991; Radford et al. 1991b); Mrk 231, with a CO source radius $0.7 \mathrm{kpc}$ (Bryant \& Scoville 1996); and 17208 - 0014, with a CO radius < $1.2 \mathrm{kpc}$ (Planesas, Mirabel, \& Sanders 1991). The small $\mathrm{CO}$ sizes and the $\mathrm{H}_{2}$ masses estimated from the CO luminosities yield column densities $n\left(\mathrm{H}_{2}\right) \geq 10^{24} \mathrm{~cm}^{-2}$. For a Galactic gas-to-dust mass ratio of $100-200$, the standard relation for far IR dust opacity (e.g., Hildebrand 1983) then yields $\tau_{\text {dust }}>1$ at $100 \mu \mathrm{m}$, so the far IR source is optically thick.

b) Tight correlation of $I_{\mathrm{CO}}$ with $100 \mu \mathrm{m}$ flux implies black body radiation. For the ultraluminous galaxies, CO integrated line intensity, $I_{\mathrm{CO}}$, is tightly correlated with $100 \mu \mathrm{m}$ flux density (Fig. 4). The scatter in this correlation is about a factor of two. As we showed earlier (DSR), this flux-flux correlation can be understood if both the CO and the dust are optically thick. Not only does CO line intensity vary linearly with $100 \mu \mathrm{m}$ flux density, but the observed constant of proportionality agrees with the black body model. To demonstrate this, the argument in Downes, Solomon, \& Radford (1993), that was given in terms of the monochromatic $100 \mu \mathrm{m}$ luminosity, $L_{100}$, will now be reformulated in terms of the total far IR luminosity $L_{\mathrm{FIR}}$. In our earlier presentation of this argument, we assumed the CO linewidth $\Delta V$ was the same on on all lines of sight; in this version, we allow for a velocity filling factor $f_{V}$. 
Assume the CO traces a huge cloud of area filling factor unity and the dust is opaque for $\lambda \leq 100 \mu \mathrm{m}$. The source may be a disk, a torus, a bar, or have an irregular shape. For illustration, we derive the luminosity ratio for a sphere, but as long as the CO and far IR sources cover the same area, the FIR/CO luminosity ratio is independent of source shape. For an optically thick sphere of temperature $T_{d}$ at its outer radius $R$,

$$
L_{\mathrm{FIR}}=4 \pi R^{2} \sigma T_{d}^{4}
$$

where $\sigma$ is the Stephan-Boltzmann constant. Similarly, the $\mathrm{CO}(1-0)$ luminosity

$$
L_{\mathrm{CO}}=4 \pi^{2} R^{2} \frac{2 k}{\lambda^{2}} \int T_{b} d \nu
$$

where $T_{b}$ is the intrinsic brightness temperature, $\nu$ is frequency, and $L_{\mathrm{CO}}$ is in the same units as $L_{\text {FIR }}$ (e.g., Watts or $\mathrm{L}_{\odot}$ ). We assume that regardless of how the gas is clumped in volume, the region has an area filling factor of unity. If $T_{b}=T_{d}$,

$$
\frac{L_{\mathrm{FIR}}}{L_{\mathrm{CO}}}=\frac{\sigma T_{d}^{3} c^{3}}{2 \pi k \nu_{\mathrm{CO}}^{3} f_{V} \Delta V},
$$

where $\nu_{\mathrm{CO}}$ is the $\mathrm{CO}(1-0)$ rest frequency and $\Delta V$ is the linewidth. For typical values,

$$
\begin{gathered}
\frac{L_{\mathrm{FIR}}}{L_{\mathrm{CO}}}=4.8 \times 10^{6}\left(\frac{T_{d}}{50 K}\right)^{3}\left(\frac{300}{f_{V} \Delta V}\right) . \\
\frac{L_{\mathrm{FIR}}}{L_{\mathrm{CO}}}=4.8 \times 10^{6}\left(\frac{L_{\mathrm{FIR}}}{10^{12} L_{\odot}}\right)^{3 / 4}\left(\frac{300 \mathrm{pc}}{R}\right)^{3 / 2}\left(\frac{300}{f_{V} \Delta V}\right) .
\end{gathered}
$$

With the CO luminosity in $\mathrm{K} \mathrm{km} \mathrm{s}^{-1} \mathrm{pc}^{2}$,

$$
L_{\mathrm{CO}}^{\prime} \equiv \pi R^{2} T_{b} f_{V} \Delta V
$$

the ratio becomes

$$
\frac{L_{\mathrm{FIR}}}{L_{\mathrm{CO}}^{\prime}}=\frac{4 \sigma T_{d}^{4}}{f_{V} \Delta V T_{b}}
$$

If $T_{b}=T_{d}$, then for typical values,

$$
\frac{L_{\mathrm{FIR}}}{L_{\mathrm{CO}}^{\prime}}=224\left(\frac{T_{d}}{50 K}\right)^{3}\left(\frac{300}{f_{V} \Delta V}\right),
$$

where the $L_{\mathrm{FIR}} / L_{\mathrm{CO}}^{\prime}$ ratio now has units of $\mathrm{L}_{\odot}\left(\mathrm{K} \mathrm{km} \mathrm{s}^{-1} \mathrm{pc}^{2}\right)^{-1}$.

Figure 5 shows this predicted ratio vs. dust temperature, for a typical $f_{V} \Delta V=300 \mathrm{~km} \mathrm{~s}^{-1}$, together with the observed ratios for ultraluminous galaxies in our sample. The median $L_{\mathrm{FIR}} / L_{\mathrm{CO}}^{\prime}=160 \mathrm{~L}_{\odot} / \mathrm{K} \mathrm{km} \mathrm{s}^{-1} \mathrm{pc}^{2}$. This is within a factor of three 
of the black body limit for temperatures $40-80 \mathrm{~K}$. Galaxies with $L_{\mathrm{FIR}}>10^{12} \mathrm{~L}_{\odot}$ are closer to the black body limit than the somewhat less luminous galaxies in our sample.

Normal spirals have $L_{\mathrm{FIR}} / L_{\mathrm{CO}}^{\prime}$ ratios 10 to 30 times lower than the black body limit in

Fig. 5. In normal spirals, the gas and dust extend over several kpc, so the column density is lower and the dust is transparent to its own radiation in the far IR. The black body limit applies if the same amount of matter is confined to a smaller volume, making the dust opaque and allowing all the dust to equilibrate with the radiation field. Ultraluminous galaxies indeed have $L_{\mathrm{FIR}} / L_{\mathrm{CO}}^{\prime}$ ratios close to the black body limit, showing their far IR dust radiation is opaque at $\lambda \leq 100 \mu \mathrm{m}$. The absence of strong near-mid IR $(25 \mu \mathrm{m})$ peaks in $90 \%$ of our sample galaxies shows opaque dust blocks our view into warmer components where the spectrum peaks in the near IR, so we see only an enormous, $\sim 300$ pc photosphere at $\sim 60 \mathrm{~K}$.

In reality, ultraluminous galaxies may differ from the black body model in the following respects:

a) $\mathrm{CO}$ brightness temperature $\neq$ dust temperature. At the mean $\mathrm{H}_{2}$ density in the central regions of ultraluminous galaxies (typically $\sim 10^{3}-10^{4} \mathrm{~cm}^{-3}$ ), the gas and dust are probably not coupled, so the gas kinetic temperature may be only about half the dust temperature. At that density, however, the brightness temperature of the $\mathrm{CO}(1-0)$ line will be close to the gas kinetic temperature. Although we took $T_{b}=T_{d}$ in our example, the argument is the same if the $\mathrm{CO}$ brightness temperature is half the dust temperature; the predicted $L_{\mathrm{FIR}} / L_{\mathrm{CO}}^{\prime}$ is twice as large, that is, even higher than the observed values (Fig. 5).

b) CO size larger than far IR size. The gas probably occupies a larger volume than the opaque dust, which may be concentrated toward H II regions. If so, the radius in eq.(5) will be larger than that in eq. (4), lowering the predicted FIR/CO luminosity ratio toward the observed data (Fig. 5). Hence, a larger CO size compensates for a lower CO temperature, so the simple black body model may give the right ratio after all.

c) Area filling factors. We assumed area filling factors of unity, but real sources have windows through the opaque dust that allow us to see near IR radiation and, in some sources, optical lines (redshifts of these objects are measured from optical lines, some of which, however, may come from gas outside of the opaque nuclear regions). Provided the area filling factors are about the same in the far IR and in CO, the black body argument will be the same, and the predicted far IR to $\mathrm{CO}$ ratio will be as in the above equations.

\section{CO SIZE AND MOLECULAR MASS}

\subsection{Radii Predicted by the Black Body Model}


The black body equation (4) sets a lower limit to the far IR source size. In a more complex model with several optically thick, massive sources, the true radius would be larger, but the radiating surface area would remain the same. For some ultraluminous galaxies, the minimum radius is about the same size as the observed nonthermal radio source (Condon et al. 1991). This far IR black body radius ( $R_{\mathrm{bb}}$ in Table 3 ) may also be compared to the minimum CO radius derived from eq. (9) with the assumptions that the dust and CO brightness temperatures are the same and the velocity filling factor $f_{V}$ is unity.

$$
R_{\mathrm{CO}}(\min )=L_{\mathrm{CO}}^{\prime} /\left(\pi T_{\mathrm{bb}} \Delta V\right)^{0.5}
$$

This is the minimum radius a CO source would have if its brightness temperature does not exceed the black body dust temperature. For the sources in our sample, the minimum CO radius (Table 3 ) is typically twice as large as the far IR black body radius, although sometimes the two agree. If these conditions are not fulfilled (e.g., a core-halo source), then the $\mathrm{CO}$ core radius scales as:

$$
R_{\mathrm{CO}}(\text { core })=R_{\mathrm{CO}}(\min )\left(\frac{1}{f_{V}}\right)^{0.5}\left(\frac{L_{\text {core }}^{\prime}}{L^{\prime}}\right)^{0.5}\left(\frac{T_{\mathrm{bb}}}{T_{b}}\right)^{0.5}
$$

The minimum observed linewidths (Table 1) are $\sim 150 \mathrm{~km} \mathrm{~s}^{-1}$, presumably from the face-on galaxies, while the larger observed linewidths suggest a true rotation velocity $V \sim 300 \mathrm{~km} \mathrm{~s}^{-1}$. The velocity filling factor $f_{V}$ would thus be $150 / 300=0.5$ for edge-on galaxies, and 1.0 for face-on galaxies. Interferometer observations of a few ultraluminous galaxies (e.g., Scoville et al. 1991) suggest $\left(L_{\text {core }}^{\prime} / L^{\prime}\right)=0.5$, and $T_{\mathrm{bb}} / T_{b}=2$. Hence on average, the true $\mathrm{CO}$ core radii may be $\sqrt{2}$ times larger than the minimum $\mathrm{CO}$ core radii listed in Table 3.

\subsection{Dynamical Mass as an Upper Limit on Gas Mass}

We calculated dynamical masses for the central regions of the ultraluminous galaxies by using the black body dust temperature derived from the $60 / 100 \mu \mathrm{m}$ flux ratio to obtain a radius for the $\mathrm{CO}$ emitting region with eq.(12). We assume the characteristic velocity $V$ in this region is determined by gravity, so the enclosed dynamical mass is $R_{\mathrm{CO}} V^{2} / G$, or

$$
M_{\mathrm{dyn}}=\left(\frac{L_{\mathrm{CO}}}{\pi T_{b} f_{V} \Delta V}\right)^{0.5} \frac{V^{2}}{G} .
$$

For a velocity filling factor $f_{V}=1$, the CO radius is a minimum radius, so a dynamical mass estimated this way is also a minimum value. As above, $T_{b}$ is the $\mathrm{CO}$ intrinsic brightness 
temperature (assumed here to be the black body dust temperature), $\Delta V$ is the observed CO linewidth (Table $\mathbf{1}$ ), and $V$ is the true velocity in the region.

In our sample, galaxy inclinations are unknown, so we estimated the true velocity $V$ statistically. The histogram of CO linewidths for the sample (Fig. 6) has a median of $300 \mathrm{~km} \mathrm{~s}^{-1}$. We therefore assumed all the galaxies in our sample had a minimum internal velocity of $300 \mathrm{kms}^{-1}$, and that those with smaller observed linewidths were inclined to our line of sight. The face-on galaxies are those with the minimum observed linewidths of $\sim 150 \mathrm{~km} \mathrm{~s}^{-1}$. To calculate minimum dynamical masses listed in Table 3 , we thus took the minimum CO core radii, with $f_{V}=1$ in eq.(9), and we took $V=\max \left(300 \mathrm{~km} \mathrm{~s}^{-1}, \Delta V\right)$. If the motions are primarily radial, then $\mathrm{V}=3^{0.5} \Delta V$.

We also list in Table 3 the equivalent total $\mathrm{H}_{2}$ density, $n_{\text {tot }}$, a maximum to the true volume-averaged $\mathrm{H}_{2}$ density, derived from the dynamical masses and the minimum $\mathrm{CO}$ radii. To calculate the minimum $\mathrm{CO}$ radii, we assumed thermalization, with the $\mathrm{CO}(1-0)$ brightness temperature equal to the gas kinetic temperature. At a kinetic temperature of $60 \mathrm{~K}$, this would occur for $\mathrm{H}_{2}$ densities $>1500 \mathrm{~cm}^{-3}$. Even if the $\mathrm{CO}$ emitting gas were to evenly fill the volume inside $R_{\mathrm{CO}}$ as an intercloud medium, the CO rotational levels would be thermalized in about half of the sources.

Since the gas mass, $M\left(\mathrm{H}_{2}\right)$, cannot exceed the dynamical mass, the average ratio $M_{\mathrm{dyn}} / L_{\mathrm{CO}}^{\prime}=1.4$ (Table 3 ) suggests that $\alpha$, the $\mathrm{H}_{2}$-mass-to-CO-luminosity ratio, may be at least three times lower than in Galactic molecular clouds. This is consistent with the black body model. For gravitationally bound clouds, this ratio is $\alpha=2.1 \cdot n^{0.5} / T_{b}$ (e.g., Radford, et al. 1991a); CO thermalization requires that $n\left(\mathrm{H}_{2}\right)=2000 \mathrm{~cm}^{-3}$ to obtain $T_{b}=60 \mathrm{~K}$, yielding $\alpha \approx 1.6 \mathrm{M}_{\odot} / \mathrm{K} \mathrm{km} \mathrm{s}^{-1} \mathrm{pc}^{2}$.

The dynamical mass listed in Table 3 is the minimum dynamical mass of a core source that has all the observed CO luminosity, $L_{\mathrm{CO}}^{\prime}$, a CO brightness temperature $T_{b}(\mathrm{CO})$ equal to the far IR black body temperature $T_{\mathrm{bb}}$, and a velocity filling factor $f_{V}=1$. If these assumptions are not fulfilled (e.g., a core-halo source), then dynamical mass scales linearly with $R_{\mathrm{CO}}$ (core) as in eq.(13). Hence to get the true dynamical mass in the CO core region, the values of $M_{\mathrm{dyn}}(\mathrm{min})$ in Table 3 should be multiplied by

$$
\frac{M_{\mathrm{dyn}}(\text { core })}{M_{\mathrm{dyn}}(\min )}=\left(\frac{1}{f_{V}}\right)^{0.5}\left(\frac{L_{\text {core }}^{\prime}}{L^{\prime}}\right)^{0.5}\left(\frac{T_{\mathrm{bb}}}{T_{b}(\mathrm{CO})}\right)^{0.5} .
$$

Similarly, the ratio $M_{\mathrm{dyn}}(\mathrm{min}) / L_{\mathrm{CO}}^{\prime}$ listed in Table 3 can be scaled by the same factor to get the true ratio of core dynamical mass to total CO luminosity:

$$
\frac{M_{\mathrm{dyn}}(\text { core })}{L_{\mathrm{CO}}^{\prime}}=\frac{M_{\mathrm{dyn}}(\min )}{L_{\mathrm{CO}}^{\prime}}\left(\frac{1}{f_{V}}\right)^{0.5}\left(\frac{L_{\mathrm{core}}^{\prime}}{L^{\prime}}\right)^{0.5}\left(\frac{T_{\mathrm{bb}}}{T_{b}(\mathrm{CO})}\right)^{0.5} .
$$

For the values suggested above, namely, $f_{V}=0.5,\left(L_{\text {core }}^{\prime} / L^{\prime}\right)=0.5$, and $T_{\mathrm{bb}} / T_{b}(\mathrm{CO})=2$, 
the true dynamical mass and the ratio $M_{\mathrm{dyn}} / L^{\prime}$ would both be $\sqrt{2}$ times higher than the values in Table 3 .

A check on the dynamical masses derived here can be provided by the fits to the $2.3 \mu \mathrm{m}$ bandhead absorption originating in stellar atmospheres to estimate the velocity dispersion of the nuclear bulge stars. In a $1.5^{\prime \prime}$ square aperture centered on NGC6240S, Lester \& Gaffney (1994) find $\sigma=350 \mathrm{~km} \mathrm{~s}^{-1}$, and estimate $M(<260 \mathrm{pc})=2 R \sigma^{2} / G=1.9 \times 10^{10} \mathrm{M}_{\odot}$. This agrees reasonably well with our measurement (Table 3), of $\Delta V=370 \mathrm{~km} \mathrm{~s}^{-1}$, and $M(<340 \mathrm{pc})=R \Delta V^{2} / G=1.1 \times 10^{10} \mathrm{M}_{\odot}$. A similar estimate has been made for Arp 220 by Shier, Rieke, \& Rieke (1994), yielding $M(<350 \mathrm{pc})=1.8 \times 10^{9} \mathrm{M}_{\odot}$, which is ten times lower than our estimate of the dynamical mass (Table 3 ). This is mainly due to the low velocity dispersion of only $125 \mathrm{~km} \mathrm{~s}^{-1}$ deduced by Shier et al., whereas we took the observed Arp $220 \mathrm{CO}$ linewidth of $480 \mathrm{~km} \mathrm{~s}^{-1}$. The large CO linewidth is also present in our millimeter spectra of $\mathrm{HCN}$ and CS. We suspect that the $2 \mu \mathrm{m}$ CO bandhead data from Arp 220 may be affected by the heavy extinction to the western near IR peak, and may not refer to the same volume as the millimeter line data.

\subsection{Lower Limit to $\mathrm{H}_{2}$ Mass: Optically Thin CO}

Since CO is always optically thick in Milky Way GMC's and spiral galaxies, we can get a useful lower limit to the $\mathrm{H}_{2}$ mass by assuming the $\mathrm{CO}(1-0)$ line has an optical depth $\tau \leq 1.0$. The minimum $\mathrm{H}_{2}$ gas mass is then

$$
M_{\text {thin }}=6.9 \times 10^{-3} M_{\odot} \quad L_{\mathrm{CO}}^{\prime} \quad T_{b}
$$

for $\quad L_{\mathrm{CO}}^{\prime}$ in $\mathrm{K} \mathrm{km} \mathrm{s}^{-1} \mathrm{pc}^{2}$ and $T_{b}$ in $\mathrm{K}$. We assume the rotational levels are thermalized and the $\mathrm{CO} / \mathrm{H}_{2}$ abundance ratio is $1.0 \times 10^{-4}$. The black body model for $T_{b}$ and the observed line luminosities yield an optically thin molecular mass given in Table 4 . They are typically $2-5 \times 10^{9} \mathrm{M}_{\odot}$, or about one-third the dynamical mass. Even if the emission is optically

thin a large fraction of the dynamical mass is $\mathrm{H}_{2}$ with $M_{\text {thin }}=0.34 \pm 0.12 M_{\text {dyn }}$. Evidence from CO excitation points to optical depths $\tau>1.0$ (Radford, et al. 1991a) indicating that a mass based on the optically thin approximation is good lower limit. In this model, if the CO optical depth is only $\sim 3$, then molecular gas alone would account for most of the dynamical mass. While the assumption of thermalized rotational levels may increase the thin mass estimate by a factor of 2 due to an overestimate of the partition function, this will be more than compensated for by the realistic optical depth. The minimum (optically thin) molecular mass would be lower only if the metallicity were significantly greater than Solar and the $\mathrm{CO} / \mathrm{H}_{2}$ abundance ratio much greater than in Milky Way GMC's. 


\subsection{Dust Mass from $100 \mu \mathrm{m}$ Emission}

An alternative method of estimating the mass of molecular gas which is independent of spectral line observations is to use the far infrared emission and assume the dust is optically thin. This is, of course, inconsistent with the black body model of optically thick dust but it provides an alternative measure of mass. To calculate the dust mass we assume the dust is thin, its emissivity varies as $\lambda^{-1.5}$, and its far IR emission coefficient is that given by Hildebrand (1983). This should be regarded as a rough estimate since IR emissivity of dust in molecular clouds is not well calibrated. The dust temperature determined from the $60 / 100 \mu \mathrm{m}$ ratio is typically $40-50 \mathrm{~K}$, and the mass is estimated from the $100 \mu \mathrm{m}$ flux. Since this measures only warm dust radiating at $100 \mu \mathrm{m}$, it is strictly a lower limit to the total dust mass. Near the center of a galaxy, however, most of the dust will indeed be warm. If we further assume the gas-to-dust mass ratio is 100, the total molecular mass can be calculated (column 4 of Table 4 ). The average gas mass estimated from the warm dust emission is very close to the dynamical mass, with $100 M_{\text {dust }}=1.1 \pm 0.5 M_{\text {dyn }}$

\subsection{Modified $\mathrm{H}_{2}$ mass-to- $\mathrm{CO}$ luminosity Relation}

For a galaxy containing virialized molecular clouds, the $\mathrm{H}_{2}$ mass-to-CO luminosity relation can be expressed as

$$
M^{\prime}\left(\mathrm{H}_{2}\right)=\alpha L_{\mathrm{CO}}^{\prime}
$$

Use of the Milky Way $\mathrm{H}_{2}$ mass-to-CO luminosity ratio, $\alpha=4.6 \mathrm{M}_{\odot} / \mathrm{K} \mathrm{km} \mathrm{s}^{-1} \mathrm{pc}^{2}$, clearly overestimates the $\mathrm{H}_{2}$ mass in ultraluminous galaxies (Table 2). We previously (DSR) related the $\mathrm{CO}$ luminosity to the dynamical mass as well as the $\mathrm{H}_{2}$ mass. This model may explain why the true gas masses are lower than the masses derived with the Milky Way ratio. The key point is that unlike Galactic clouds or gas distributed in the disks of galaxies, the $\mathrm{CO}$ in the centers of ultraluminous galaxies may not come from virialized clouds, but from a filled intercloud medium, so the linewidth is determined by the total dynamical mass in the region (gas and stars), that is, $\Delta V^{2}=G M_{\mathrm{dyn}} / R$. The CO line emission may trace a medium bound by the total potential of the galactic center, containing a mass $M_{\mathrm{dyn}}$ consisting of stars, dense clumps, and an interclump medium containing the $\mathrm{CO}$ emitting gas with mass $M\left(\mathrm{H}_{2}\right)$.

Defining $f \equiv M$ (gas) $/ M_{\text {dyn }}$, we showed the usual CO to $\mathrm{H}_{2}$ mass relation becomes

$$
\begin{aligned}
& M_{\mathrm{dyn}} / L_{\mathrm{CO}}^{\prime}=f^{-1 / 2} \alpha=f^{-1 / 2} 2.6(\bar{n})^{1 / 2} T_{\mathrm{b}}^{-1} \\
& M\left(\mathrm{H}_{2}\right) / L_{\mathrm{CO}}^{\prime}=f^{1 / 2} \alpha=f^{1 / 2} 2.6(\bar{n})^{1 / 2} T_{\mathrm{b}}^{-1}
\end{aligned}
$$


and

$$
M_{\mathrm{dyn}} M\left(\mathrm{H}_{2}\right)=\left(\alpha L_{\mathrm{CO}}^{\prime}\right)^{2},
$$

where $\bar{n}$ is the $\mathrm{H}_{2}$ density averaged over the whole volume. We argued in our earlier paper that the quantity $\alpha L_{\mathrm{CO}}^{\prime}$ measures the geometric mean of total mass and gas mass. It underestimates total mass and overestimates gas mass. Hence if the $\mathrm{CO}$ emission in ultraluminous galaxies comes from regions not confined by self gravity, but instead from an intercloud medium bound by the potential of the galaxy, or from molecular gas in pressure, rather than gravitational, equilibrium, then the usual relation $M\left(\mathrm{H}_{2}\right) / L_{\mathrm{CO}}^{\prime}=\alpha$ must be changed to eq.(20).

\subsection{Summary of Revised Mass Estimates}

For the compact central regions, the dynamical mass is the best estimator of the molecular mass. The dynamical masses listed in Table 4 are those for the black-body model; the actual dynamical mass is given by eq.(15). We have argued here that the black-body model is a good approximation for ultraluminous galaxies since it comes close to predicting the ratio of far IR to $\mathrm{CO}$ luminosity. The $\mathrm{CO}$ luminosity to molecular mass conversion factor for ultraluminous galaxies must be $\leq M_{\mathrm{dyn}} / L_{\mathrm{CO}}^{\prime} \sim 1.4$. is given in Table 3). Our estimates of the gas mass from optically thin $\mathrm{CO}(1-0)$ (Column 3 of Table 4) and from optically thin dust (Column 4 of Table 4) indicate that a large fraction of the dynamical mass is molecular gas (i.e., $f$ approaches 1 in equations 19 and 20). If the dynamical mass is dominated by the gas mass and the core size is close to that of the black-body model then

$$
M\left(\mathrm{H}_{2}\right)=1.0 \times 10^{10} M_{\odot},
$$

with a dispersion of only $0.3 \times 10^{10} \mathrm{M}_{\odot}$. This is about three times lower than previous estimates of the gas mass which have utilized the Milky Way $M\left(\mathrm{H}_{2}\right) / L_{\mathrm{CO}}^{\prime}$ ratio. Even if the velocity filling factor is 0.5 and the excitation temperature half that of the dust the dynamical mass is only increased by a factor of 2 . More accurate determinations of the dynamical mass will come from interferometer measurements of the $\mathrm{CO}$ radii for the nearer galaxies in the survey.

The standard $\mathrm{M}^{\prime}\left(\mathrm{H}_{2}\right) / L_{\mathrm{CO}}^{\prime}=4.6 \mathrm{M}_{\odot} / \mathrm{K} \mathrm{km} \mathrm{s}^{-1} \mathrm{pc}^{2}($ Column 2 of Table 2), appropriate for the molecular gas in a more typical galactic environment outside the central core, is an overestimate for ultraluminous galaxies. 


\section{PAIR SEPARATION AND $L_{\mathrm{FIR}} / L_{\mathrm{CO}}$ OF ULTRALUMINOUS GALAXIES}

The prevalence of close interactions among IR bright galaxies was noted soon after the first IRAS galaxy catalog became available (Lonsdale, Persson, \& Matthews 1984; Soifer et al. 1984). In Fig. 7 we present R-band CCD images of 12 sample galaxies between RA 10h to $23 \mathrm{~h}$ (Sanders \& Kim, private communication); they show a wide variation in morphology and pair separation. Some appear distinctly single, with only slightly distorted disks, e.g., 1609-0139, while others are prominent, closely interacting galaxies with two disks and two nuclei, e.g., $10190+1322$ or $15030+4835$. One of the most luminous and most distant objects in our sample, $14070+0525$, appears to be an isolated galaxy, although very faint tails would be invisible on this image. Table 5 summarizes the data on pair separation for the sample, from the literature and from Fig. 7 . The measured separations range from 0.3 to $14 \mathrm{kpc}$ with an average of $6 \mathrm{kpc}$. Near IR images of some close ultraluminous galaxies such as Arp 220 and Mrk 231 show that galaxies apparently single in optical images may have double nuclei with small separations, indicating a nearly completed merger (Graham et al. 1990; Majewski et al. 1993; Armus et al. 1994). Conversely some galaxies which appear double in optical images clearly have only one nucleus in $\mathrm{K}$ band images (Murphy et al. 1996). Given the large amount of dust in these objects, however, even the near IR images may be heavily affected by extinction, and suspected double nuclei may actually be bright objects outside of the heavily obscured CO-emitting zone.

On the basis of R band images of ten nearby ultraluminous galaxies, Sanders et al. (1988a) argued that all ultraluminous galaxies are strongly interacting, as shown by tidal tails, rings or double nuclei with separations $<5 \mathrm{kpc}$, and that the fraction of close doubles increases with IR luminosity. From optical morphology and spectra, they suggested ultraluminous galaxies represent the initial dust enshrouded stage in the formation of quasars. In their model, most of the luminosity comes from an AGN rather than star formation. Because very few of the ultraluminous galaxies found by IRAS have true quasar or Seyfert 1 spectra, Sanders et al. argued the AGN was completely hidden by dust and the transition phase to a dust-free AGN is represented by the "warm" ultraluminous galaxies with $S_{25} / S_{60}>0.2$ (Sanders et al. 1988b). Only five of the objects in our sample have $S_{25} / S_{60}>0.2$ and four of these overlap with Sanders et al.'s sample. However, the object with the highest $L_{\mathrm{FIR}} / L_{\mathrm{CO}}^{\prime}, 08572+3915$, is a warm galaxy but is definitely not a completed merger since its separation is $5.5 \mathrm{kpc}$ (Sanders 1992).

There are no significant correlations of pair separation (Table 5) with CO luminosity or more importantly the ratio of far IR to CO luminosity, $L_{\mathrm{FIR}} / L_{\mathrm{CO}}^{\prime}$. The apparent stage of the interaction appears to have little effect on the efficiency of star formation or the fueling of the putative black hole. Extremely luminous infrared galaxies include completed mergers 
and interacting pairs with separations of approximately 10kpc. Two of the more distant and more luminous objects in our sample, $16334+4630$ and $15030+4835$, with very cool far IR colors, have large pair separations, 11 and $12 \mathrm{kpc}$ (Fig. 7) respectively. Both have $L_{\mathrm{FIR}} \approx 2 \times 10^{12} \mathrm{~L}_{\odot}$, similar to Mrk 231 or the quasar Mrk 1014. If an evolutionary sequence is present in ultraluminous galaxies it is not apparent from the CO or IR data combined with the optical morphology. 


\section{DISCUSSION}

\subsection{Dense Molecular Gas}

In addition to a high $\mathrm{IR} / \mathrm{CO}$ luminosity ratio, the molecular gas in the centers of ultraluminous galaxies exhibits other differences from GMC's in the Milky Way and normal spiral galaxies. Most important is the extremely high HCN luminosity. HCN emission traces $\mathrm{H}_{2}$ at a much higher density, $\sim 10^{5} \mathrm{~cm}^{-3}$, than $\mathrm{CO}\left(\sim 500 \mathrm{~cm}^{-3}\right)$. As we have shown previously, the HCN luminosities of the ultraluminous galaxies Mrk 231, Arp 220, and NGC 6240 are greater than the CO luminosity of the Milky Way (Solomon, Downes, \& Radford 1992a). The ratio of $\mathrm{HCN}$ to $\mathrm{CO}$ luminosity is $1 / 4$ to $1 / 7$ for ultraluminous galaxies, but only $1 / 30$ or less in the disks of normal spiral galaxies. This implies that a large fraction of the molecular gas in ultraluminous galaxies, perhaps $50 \%$, is in very dense regions similar to star forming cloud cores in Orion or W51, rather than in the envelopes of giant molecular clouds, as is the case in the disks of normal spiral galaxies.

If galactic HCN luminosity is taken as a dense gas indicator and far infrared luminosity as a high mass star formation indicator, then the star formation rate per mass of dense gas is the same for normal spirals and IR luminous interacting galaxies. There is sufficient dense gas in ultraluminous galaxies to account for their luminosity by star formation. In our model, the $\mathrm{CO}$ emission from ultraluminous galaxies originates from an intercloud medium essentially filling the volume; the emission from HCN and CS is from the dense "clouds" embedded inside the molecular region. The entire ISM is a scaled up version of a normal galactic disk. This environment is an ideal stellar nursery for prodigious star formation leading to ultraluminous galaxies.

\subsection{Summary of $\mathrm{CO}$ Observations and $\mathrm{H}_{2}$ Mass}

Of the 37 galaxies in the sample, 32 have a far infrared luminosity greater than $6.5 \times 10^{11} \mathrm{~L}_{\odot}$ and total IR luminosities greater than $8 \times 10^{11} \mathrm{~L}_{\odot}$. We refer to these as ultraluminous IR galaxies. They share several properties in common:

1. All but one of the ultraluminous galaxies have a high $\mathrm{CO}(1-0)$ luminosity, with values of $\log \left(L_{\mathrm{CO}}^{\prime} / \mathrm{K} \mathrm{km} \mathrm{s}^{-1} \mathrm{pc}^{2}\right)=9.92 \pm 0.12$. The extremely small dispersion of only 30 $\%$ is half that of the far infrared luminosity. The parameter with the narrowest range (best defined) is not IR luminosity but CO luminosity, even though the selection was based on IR luminosity. 
2. We have demonstrated that for ultraluminous galaxies in which the central few hundred parsecs is dominated by a molecular core, the CO luminosity and the ratio of FIR to CO luminosity can be explained as a consequence of thermal emission from optically thick dust and $\mathrm{CO}$ in a central sphere, torus (ring) or disk. The ambient densities are a factor of 100 higher than in a galactic disk, making even the intercloud medium molecular throughout. The median value of $L_{\mathrm{FIR}} / L_{\mathrm{CO}}^{\prime}=$ $160 \mathrm{~L}_{\odot} / \mathrm{K} \mathrm{km} \mathrm{s}^{-1} \mathrm{pc}^{2}$, within a factor of 2 of the black body limit for the observed far IR temperatures.

3. From the observed linewidths, we estimate the dynamical mass within the minimum CO radius $(\sim 400 \mathrm{pc})$. This provides an upper limit on the mass of molecular gas. For a velocity filling factor of 1.0 , the dynamical mass is $1.0 \pm 0.3 \times 10^{10} \mathrm{M}_{\odot}$. A more likely velocity filling factor of 0.5 raises the upper limit to the mass by $2^{0.5}$.

4. We assume the $\mathrm{CO}$ is optically thin in the (1-0) line to estimate the gas mass from the observed CO luminosity. This provides a lower limit on the mass of molecular gas of typically $2-5 \times 10^{9} \mathrm{M}_{\odot}$, with $M_{\text {thin }}=0.34 \pm 0.12 M_{\text {dyn }}$ or about one-third the dynamical mass.

5. We assume the dust is optically thin at $100 \mu \mathrm{m}$ to estimate the minimum mass of interstellar material. This provides another, independent measure of the mass of molecular gas. The average gas mass estimated from the dust continuum emission assuming a dust to gas ratio of 100 is very close to the dynamical mass, $100 M_{\text {dust }}=1.1 \pm 0.5 M_{\text {dyn }}$.

6. The standard Milky Way ratio, $M^{\prime}\left(\mathrm{H}_{2}\right) / L_{\mathrm{CO}}^{\prime}=4.6 \mathrm{M}_{\odot}\left(\mathrm{K} \mathrm{km} \mathrm{s}^{-1} \mathrm{pc}^{2}\right)^{-1}$, yields a mass $M^{\prime}\left(\mathrm{H}_{2}\right)=3.7 M_{\mathrm{dyn}}$, which is clearly an overestimate. We show that in the extreme environment near the center of an ultraluminous galaxy, where the CO emission originates from an intercloud medium which essentially fills a volume rather than from clouds bound by self gravity, the CO luminosity traces the geometric mean of the molecular mass and the total dynamical mass. The true total molecular mass must be between the optically thin $\mathrm{CO}$ estimate and the dynamical mass. The agreement between the total gas mass estimated from the dust emission and the dynamical mass suggests a number closer to the dynamical mass. Dynamical mass estimates with sizes determined from interferometer measurements are required to obtain better estimates of the molecular mass in ultraluminous galaxies. 


\subsection{The Effect of Close Interactions on the Molecular ISM}

Molecular clouds are in virial equilibrium, not pressure equilibrium, (eg. Solomon et al., 1987) maintained by a balance between self gravity and turbulence driven primarily by star formation. The external pressure from the ambient ISM or intercloud medium is significantly less than the internal effective pressure characterized by supersonic velocities. The star formation is self regulating and GMC's in galactic disks have modest star formation rates per solar mass ( Mooney and Solomon, 1988).

The uniformly high CO luminosities found here for all ultraluminous galaxies, combined with the evidence of very large quantities of even denser molecular gas, show that the molecular gas required for an extreme starburst is always present when a galaxy has ultrahigh infrared luminosity. Does this imply that a merger or close interaction is the ideal site for the transformation of diffuse gas into molecular clouds or the stimulation of high mass star formation by cloud collisions (eg. Scoville, Sanders \& Clemens, 1986)? Both seem highly unlikely given the typical interaction velocity of 200 or $300 \mathrm{~km} / \mathrm{s}$, more than an order of magnitude greater than the escape velocity of material in even the most massive GMC's, and more than sufficient to completely ionize and destroy any trace of molecules in a direct collision between clouds. The molecular gas in luminous mergers must be from preexisting molecular clouds. Unlike diffuse gas, the mean free path for molecular clouds is sufficiently long $(\sim 2 \mathrm{kpc}$ ) that a merger, unless it is completely edge on, will result in few direct collisions. Molecular clouds can therefore survive a close interaction or merger, although they will be changed by the new environment.

Two mechanisms have been proposed for triggering an intense burst of star formation in preexisting molecular clouds during a close interaction or merger. During a direct collision the HI clouds will collide, forming a hot ionized high pressure remnant gas ( Jog and Solomon, 1992). The overpressure due to this hot gas causes a radiative shock compression of the outer layers of GMC's in the overlapping wedge region. The outer layers become gravitationally unstable, leading to a burst of star formation in the initially stable GMC's. This scenario probably applies to luminous $\left(\sim 10^{11} \mathrm{~L}_{\odot}\right)$ IR galaxies with prominent extranuclear starbursts such as Arp 299, but is not strong enough to produce ultraluminous galaxies. A more efficient mechanism for producing a central starburst in interacting galaxy pairs from preexisting clouds has been suggested by Jog and Das (1992). In this model, as a disk GMC tumbles into the central regions of a galaxy following an encounter, it undergoes radiative shock compression by the high pressure of the central molecular intercloud medium. When the growth time for the gravitational instabilities in the shocked outer shell of a cloud becomes smaller than the shock crossing time, the shell becomes unstable, resulting in a burst of star formation. The infall may be helped by the establishment of a nonaxisymmetric bar potential (eg. Norman , 1991). The luminosity 
depends on the compressed mass fraction, cloud infall rate and efficiency of star formation; evolved mergers generate a luminosity comparable to that of ultraluminous IR galaxies.

Our analysis of $\mathrm{CO}$ emission from ultraluminous galaxies reduces the $\mathrm{H}_{2}$ mass from previous estimates of $2-5 \times 10^{10} \mathrm{M}_{\odot}$ to $0.4-1.0 \times 10^{10} \mathrm{M}_{\odot}$, which is in the range found for molecular gas rich spiral galaxies. Thus a collision involving a molecular gas rich spiral could lead to an ultraluminous galaxy powered by central starbursts triggered by the compression of preexisting GMC's.

We thank Mr. Yu Gao, Stony Brook, for help with Table 5, and Fred Seward, Elizabeth Bohlen, and the Center for Astrophysics for the use of their measuring engine. We especially thank D. B. Sanders and D. C. Kim for letting us use their R band CCD images. 


\section{REFERENCES}

Armus, L., Heckman, T.M., \& Miley, G.K. 1987, AJ, 94, 831

Armus, L., Heckman, T.M., \& Miley, G.K. 1990, ApJ, 364, 471

Armus, L., Surace, J.A., Soifer, B.T., Matthews, K., Graham, J.R., \& Larkin, J.E. 1994, AJ, 108, 76

Barvainis, R., Alloin, D., \& Antonucci, R. 1989, ApJ, 337, L69

Beichman, C.A., Neugebauer, G., Habing, H.J., Clegg, P.E., Chester, T.J. (eds.) 1988, IRAS Catalogs and Atlases Explanatory Supplement, (Washington, D.C.: U.S. Govt. Printing Office)

Bryant, P.M., \& Scoville, N.Z. 1996, ApJ, 457, 692

Condon, J.J., Huang, Z.P., Yin, Q.F., \& Thuan, T.X. 1991, ApJ, 378, 65

Downes, D., Solomon, P.M., \& Radford, S.J.E. 1993, ApJ, 414, L13 (DSR).

Fisher, K.B., Huchra, J.P., Strauss, M.A., Davis, M., Yahil, A., \& Schlegel, D. 1995, ApJS, 100,69

Graham, J.R., Carico, D.P., Matthews, K., Neugebauer, G., Soifer, B.T., \& Wilson, T.D. 1990, ApJ, 354, L5

Hildebrand, R.H. 1983, QJRAS, 24, 267

Infrared Astronomical Satellite (IRAS) Point Source Catalog, Version 2 1988, Joint IRAS Science Working Group, (Washington, D.C.: U.S. Govt. Printing Office)

Jog, C.J., \& Solomon, P.M. 1992, ApJ, 387, 152

Jog, C.J., \& Das, M. 1992, ApJ, 400, 476

Kollatschny, W., Dietrich, M., Borgeest, U., \& Schramm, K.J. 1991, A\&A, 249, 57

Lester, D.F., \& Gaffney, N.I. 1994, ApJ, 431, L13

Lonsdale, C.J., Persson, S.E., \& Matthews, K. 1984, ApJ, 287, 95

Lonsdale, C.J., Helou, G., Good, J.C., \& Rice, W. 1985, in Cataloged Galaxies and Quasars Observed in the IRAS Survey, (Pasadena: JPL)

Majewski, S.R., Hereld, M., Koo, D.C., Illingworth, G.D., \& Heckman, T.M. 1993, ApJ, 402,76

Melnick, J. \& Mirabel, I.F. 1990, A\&A, 231, L19

Mihos, J.C., \& Hernquist, L. 1994, ApJ, 431, L9 
Mooney, T. \& Solomon, P.M. 1988, ApJ, 334, L51

Moshir et al. 1992, IRAS Faint Source Survey Explanatory Supplement, Version 2, (Pasadena: JPL)

Murphy, T.W. Jr., Armus L., Matthews, K., Soifer, B.T., Mazzarella, J.M., \& Shupe, D.L. 1996, AJ, 111, 1025

Norman,. C. A. 1991, in Massive Stars in Starbursts, ed. C. Leitherer, N.R. Walborn, T.M. Heckman, \& C.A. Norman, (Cambridge: Camb. Univ. Press) 271

Okumura, S.K., Kawabe, R., Ishiguro, M., Kasuga, T., Morita, K.I., \& Ishizuki, S. 1991, in Dynamics of Galaxies and Their Molecular Cloud Distributions, ed. F. Combes \& F. Casoli (Dordrecht: Kluwer), 425

Planesas, P., Mirabel, I.F., \& Sanders, D.B. 1991, ApJ, 370, 172

Radford, S.J.E., Solomon, P.M., \& Downes, D. 1991a, ApJ, 368, L15

Radford, S.J.E., et al. 1991b, in Dynamics of Galaxies and Their Molecular Cloud Distributions, ed. F. Combes \& F. Casoli (Dordrecht: Kluwer), 303

Sanders, D.B. 1992, in Relationships Between Active Galactic Nuclei and Starburst Galaxies, ASP Conf. Ser. 31, ed. A.V. Filippenko, (San Francisco: ASP) 303

Sanders, D.B., Soifer, B.T., Elias, J.H., Madore, B.F., Matthews, K., Neugebauer, G., \& Scoville, N.Z. 1988a, ApJ, 325, 74

Sanders, D.B., Soifer, B.T., Elias, J.H., Neugebauer, G., \& Matthews, K. 1988b, ApJ, 328, L35

Sanders, D.B., Scoville, N.Z., \& Soifer, B.T. 1991, ApJ, 370, 158

Sanders, D.B., et al., 1986, ApJ, 305, L45

Scoville, N.Z., Sanders, D.B., \& Clemens, D.P., 1986, ApJ, 310, L77

Scoville, N.Z., Sargent, A.I., Sanders, D.B., \& Soifer, B.T. 1991, ApJ, 366, L5

Shaya, E.J., Dowling, D.M., Currie, D.G., Faber, S.M., \& Groth, E.J. 1994, AJ, 107, 1675

Shier, L.M., Rieke, M.J., \& Rieke, G.H. 1994, ApJ, 433, L9

Soifer, B.T., et al. 1984, ApJ, 278, L71

Solomon, P.M., Downes, D., \& Radford, S.J.E. 1992a, ApJ, 387, L55

Solomon, P.M., Radford, S.J.E., \& Downes, D. 1990, ApJ, 348, L53

Solomon, P.M., Radford, S.J.E., \& Downes, D. 1992, Nature, 356, 318

Solomon, P.M., \& Rivolo, A.R. 1989, ApJ, 339, 919 
Solomon, P.M., Rivolo, A.R., Barrett, J.W., \& Yahil, A. 1987, ApJ, 319, 730

Solomon, P.M., \& Sage, L.J. 1988, ApJ, 334, 613

Strauss, M.A., Huchra, J.P., Davis, M., Yahil, A., Fisher, K.B., \& Tonry, J. 1992, ApJS, 83, 29

Wright, G.S., Joseph, R. D. \& Meikle, W.P. 1984, Nature, 344417

Weinberg, S. 1972, Gravitation and Cosmology (New York: Wiley) 
Table 1. DATA FROM THE IRAM 30 m TELESCOPE ON CO(1-0) IN ULTRALUMINOUS GALAXIES

\begin{tabular}{|c|c|c|c|c|c|c|}
\hline $\begin{array}{c}\text { Source } \\
\text { name }\end{array}$ & $\begin{array}{l}\text { Position } \\
\text { R.A. } 1950 \\
\left(\begin{array}{lll}h & \mathrm{~m} & \mathrm{~s}\end{array}\right)\end{array}$ & $\begin{array}{c}\text { Observed } \\
\text { Dec. } 1950 \\
\left(\begin{array}{ccc}\circ & \prime & \prime \prime\end{array}\right)\end{array}$ & $\begin{array}{l}\text { Redshift } \\
c z \\
\left(\mathrm{~km} \mathrm{~s}^{-1}\right)\end{array}$ & $\begin{array}{c}\text { Linewidth } \\
\Delta V \\
\left(\mathrm{~km} \mathrm{~s}^{-1}\right)\end{array}$ & $\begin{array}{c}\text { Intensity } \\
I_{\mathrm{CO}} \\
\left(\mathrm{K} \mathrm{km} \mathrm{s}^{-1}\right)\end{array}$ & $\begin{array}{c}\mathrm{CO} / \mathrm{FIR} \\
I_{\mathrm{CO}} / S_{100 \mu \mathrm{m}} \\
\left(\mathrm{Kkm} \mathrm{s}^{-1} \mathrm{Jy}^{-1}\right)\end{array}$ \\
\hline $00057+4021$ & 000545.1 & +402114 & 13390 & 350 & 9.9 & 2.30 \\
\hline $00188-0856$ & 001853.7 & -085607 & 38530 & 390 & 2.2 & 0.65 \\
\hline $00262+4251$ & 002612.9 & +425140 & 29153 & 230 & 3.5 & 1.43 \\
\hline $\mathrm{I} \mathrm{Zw}_{\mathrm{w}} 1$ & 005057.8 & +122519 & 18330 & 410 & $7.0^{b)}$ & 2.66 \\
\hline Mrk 1014 & 015716.6 & +000907 & 48947 & 200 & 1.8 & 0.83 \\
\hline $02483+4302$ & 024820.4 & +430256 & 15419 & 250 & 5.7 & 0.82 \\
\hline $03158+4227$ & 031552.3 & +422736 & 40296 & 180 & 2.1 & 0.49 \\
\hline $03521+0028$ & 035207.8 & +002820 & 45530 & 150 & 2.2 & 0.57 \\
\hline $04232+1436$ & 042315.2 & +143653 & 23855 & 400 & 7.5 & 1.76 \\
\hline VII Zw 31 & 050817.5 & +793640 & 16260 & 200 & 21.0 & 2.18 \\
\hline $07598+6508$ & 075953.0 & +650821 & $44621^{a)}$ & 337 & $2.8^{a)}$ & 1.62 \\
\hline $08030+5243$ & 080301.5 & +524345 & 25031 & 420 & 6.7 & 1.53 \\
\hline $08572+3915$ & 085713.0 & +391539 & 17450 & 270 & 2.0 & 0.44 \\
\hline $09320+6134$ & 093204.7 & +613437 & 11785 & 350 & 15.6 & 0.77 \\
\hline $10035+4852$ & 100335.5 & +485225 & 19427 & 250 & 8.8 & 1.41 \\
\hline $10190+1322$ & 101901.4 & +132204 & 22953 & 390 & 7.4 & 1.33 \\
\hline $10495+4424$ & 104930.1 & +442446 & 27674 & 330 & 5.1 & 0.94 \\
\hline $10565+2448$ & 105635.4 & +244843 & 12923 & 300 & 15.7 & 1.04 \\
\hline $11506+1331$ & 115039.8 & +133105 & 38158 & 290 & 2.5 & 0.75 \\
\hline Mrk 231 & 125405.0 & +570839 & 12650 & 230 & 22.0 & 0.73 \\
\hline $13106-0922$ & 131037.3 & -092215 & 52290 & 200 & 1.7 & 0.70 \\
\hline Arp 193 & 131817.0 & +342407 & 7000 & 410 & 36.0 & 1.43 \\
\hline Mrk 273 & 134251.6 & +560814 & 11324 & 300 & 19.0 & 0.89 \\
\hline $13442+2321$ & 134418.0 & +232114 & 42620 & 140 & 1.4 & 0.62 \\
\hline $14070+0525$ & 140700.5 & +052541 & 79621 & 270 & 0.8 & 0.42 \\
\hline
\end{tabular}


Table 1 - Continued

\begin{tabular}{|c|c|c|c|c|c|c|}
\hline $\begin{array}{l}\text { Source } \\
\text { name }\end{array}$ & $\begin{array}{l}\text { Position } \\
\text { R.A. } 1950 \\
\left(\begin{array}{lll}h & \text { m }\end{array}\right)\end{array}$ & $\begin{array}{l}\text { Observed } \\
\text { Dec. } 1950 \\
\left(\begin{array}{ccc}\circ & \prime\end{array}\right)\end{array}$ & $\begin{array}{l}\text { Redshift } \\
c z \\
\left(\mathrm{~km} \mathrm{~s}^{-1}\right.\end{array}$ & $\begin{array}{l}\text { inewidth } \\
\Delta V \\
\left(\mathrm{~km} \mathrm{~s}^{-1}\right)\end{array}$ & $\begin{array}{c}\text { Intensity } \\
I_{\mathrm{CO}} \\
\left(\mathrm{K} \mathrm{km} \mathrm{s}^{-1}\right)\end{array}$ & $\begin{array}{c}\mathrm{CO} / \mathrm{FIR} \\
I_{\mathrm{CO} / S_{100 \mu \mathrm{m}}} \\
\left(\mathrm{Kkm} \mathrm{s}^{-1} \mathrm{Jy}^{-1}\right.\end{array}$ \\
\hline $15030+4835$ & 150301.3 & +483524 & 64900 & 270 & 1.5 & 1.03 \\
\hline $\operatorname{Arp} 220$ & 153246.9 & +234008 & 5450 & 480 & 109.0 & 0.97 \\
\hline 16090-0139 & 160904.9 & -013925 & 40044 & 300 & 3.7 & 0.76 \\
\hline $16334+4630$ & 163324.3 & +463058 & 57250 & 320 & 1.5 & 0.69 \\
\hline NGC 6240 & 165027.2 & +022858 & 7298 & 370 & 69.0 & 2.48 \\
\hline $17208-0014$ & 172048.2 & -001417 & 12837 & 360 & 20.0 & 0.57 \\
\hline $18368+3549$ & 183649.5 & +354936 & 34850 & 330 & 3.4 & 0.89 \\
\hline $19297-0406$ & 192943.1 & -040624 & 25700 & 300 & 6.8 & 0.88 \\
\hline $19458+0944$ & 194552.0 & +094430 & 29980 & 350 & 6.4 & 0.90 \\
\hline $20087-0308$ & 200846.4 & -030852 & 31688 & 400 & 7.7 & 1.18 \\
\hline $22542+0833$ & 225411.3 & +083322 & 49750 & 150 & 1.2 & 0.81 \\
\hline $23365+3604$ & 233632.3 & +360434 & 19330 & 310 & 10.7 & 1.28 \\
\hline median: & - & - & & 300 & - & 0.9 \\
\hline std. dev. & - & - & & 85 & - & 0.6 \\
\hline
\end{tabular}

${ }^{a)}$ Sanders et al. (1988b), $I_{\mathrm{CO}}$ corrected by us; ${ }^{b}$ Barvainis et al. (1989).

Errors: $c z: \pm 20 \mathrm{~km} \mathrm{~s}^{-1} ; \Delta V: \pm 30 \mathrm{~km} \mathrm{~s}^{-1} ; I_{\mathrm{CO}}: \pm 20 \%$ for $I>6, \pm 35 \%$ for $2<I<6$. Linewidths are Gaussian fit values (FWHP), or else half the full width to zero intensity for non-gaussian lines (See Figure 2 for spectra).

For point sources, $S / T_{\mathrm{mb}}=4.5 \mathrm{Jy} / \mathrm{K}$ at the $30 \mathrm{~m}$ telescope at $3 \mathrm{~mm}$. 
Table 2. CO and far IR Luminosities of Ultraluminous Galaxies

\begin{tabular}{|c|c|c|c|c|c|c|c|c|c|}
\hline Source & $L_{\mathrm{CO}}^{\prime}$ & $M^{\prime}\left(\mathrm{H}_{2}\right)$ & $S_{25}$ & $S_{60}$ & $S_{100}$ & $\frac{S_{60}}{S_{100}}$ & $L_{\mathrm{FIR}}$ & $\frac{L_{\mathrm{FIR}}}{M^{\prime}\left(\mathrm{H}_{2}\right.}$ & $=\frac{L_{\mathrm{FIR}}}{L_{\mathrm{CO}}^{\prime}}$ \\
\hline name & $\left(10^{9} \mathrm{~L}_{l}\right)$ & $\left(10^{10} \mathrm{M}_{\odot}\right)$ & (Jy) & (Jy) & $(\mathrm{Jy})$ & & $\left(10^{12} \mathrm{~L}_{\odot}\right)$ & $\frac{\mathrm{L}_{\odot}}{\mathrm{M}_{\odot}}$ & $\frac{\mathrm{L}_{\odot}}{\mathrm{L}_{l}}$ \\
\hline $00057+4021$ & 3.8 & 1.7 & 0.36 & 4.47 & 4.30 & 1.04 & 0.31 & 17 & 80 \\
\hline $00188-0856$ & 6.7 & 3.1 & 0.37 & 2.59 & 3.40 & 0.76 & 1.51 & 48 & 224 \\
\hline $00262+4251$ & 6.2 & 2.9 & 0.33 & 2.98 & 2.44 & 1.22 & 0.99 & 34 & 160 \\
\hline I Zw 1 & 5.0 & 2.3 & 1.21 & 2.24 & 2.63 & 0.85 & 0.29 & 12 & 57 \\
\hline Mrk 1014 & 8.7 & 4.0 & 0.54 & 2.22 & 2.16 & 1.03 & 2.14 & 53 & 245 \\
\hline $02483+4302$ & 2.9 & 1.3 & 0.19 & 4.02 & 6.92 & 0.58 & 0.41 & 30 & 142 \\
\hline $03158+4227$ & 7.0 & 3.2 & 0.45 & 4.26 & 4.28 & 1.00 & 2.75 & 85 & 392 \\
\hline $03521+0028$ & 9.3 & 4.3 & 0.23 & 2.64 & 3.84 & 0.69 & 2.26 & 52 & 242 \\
\hline $04232+1436$ & 9.0 & 4.1 & $<0.38$ & 3.45 & 4.26 & 0.81 & 0.75 & 18 & 83 \\
\hline VII Zw 31 & 11.8 & 5.4 & 0.58 & 5.58 & 9.62 & 0.58 & 0.64 & 11 & 53 \\
\hline $07598+6508$ & 11.4 & 5.2 & 0.53 & 1.69 & 1.73 & 0.98 & 1.34 & 25 & 118 \\
\hline $08030+5243$ & 8.8 & 4.1 & 0.18 & 2.99 & 4.39 & 0.68 & 0.75 & 18 & 85 \\
\hline $08572+3915$ & 1.3 & 0.6 & 1.70 & 7.43 & 4.59 & 1.62 & 1.00 & 166 & 766 \\
\hline $09320+6134$ & 4.7 & 2.1 & 1.03 & 11.54 & 20.23 & 0.57 & 0.69 & 32 & 148 \\
\hline $10035+4852$ & 7.0 & 3.2 & 0.28 & 4.59 & 6.24 & 0.74 & 0.67 & 20 & 94 \\
\hline $10190+1322$ & 8.2 & 3.8 & 0.38 & 3.35 & 5.57 & 0.60 & 0.75 & 19 & 91 \\
\hline $10495+4424$ & 8.2 & 3.8 & 0.16 & 3.53 & 5.41 & 0.65 & 1.12 & 29 & 136 \\
\hline $10565+2448$ & 5.6 & 2.6 & 1.14 & 12.12 & 15.13 & 0.80 & 0.76 & 29 & 135 \\
\hline $11506+1331$ & 7.5 & 3.4 & 0.19 & 2.58 & 3.32 & 0.78 & 1.47 & 42 & 196 \\
\hline Mrk 231 & 7.5 & 3.5 & 8.66 & 31.99 & 30.29 & 1.06 & 1.96 & 56 & 259 \\
\hline $13106-0922$ & 9.4 & 4.3 & $<0.36$ & 1.66 & 2.42 & 0.69 & 1.89 & 43 & 201 \\
\hline Arp 193 & 3.8 & 1.8 & 1.36 & 15.44 & 25.18 & 0.61 & 0.31 & 17 & 81 \\
\hline Mrk 273 & 5.1 & 2.3 & 2.28 & 21.74 & 21.38 & 1.02 & 1.06 & 43 & 202 \\
\hline $13442+2321$ & 5.2 & 2.4 & 0.11 & 1.62 & 2.26 & 0.72 & 1.18 & 49 & 227 \\
\hline $14070+0525$ & 9.5 & 4.4 & 0.19 & 1.45 & 1.82 & 0.80 & 3.80 & 87 & 401 \\
\hline
\end{tabular}


Table 2-Continued

\begin{tabular}{|c|c|c|c|c|c|c|c|c|c|}
\hline $\begin{array}{l}\text { Source } \\
\text { name }\end{array}$ & $\begin{array}{c}L_{\mathrm{CO}}^{\prime} \\
\left(10^{9} \mathrm{~L}_{l}\right)\end{array}$ & $\begin{array}{c}M^{\prime}\left(\mathrm{H}_{2}\right) \\
\left(10^{10} \mathrm{M}_{\odot}\right)\end{array}$ & $(\mathrm{Jy})$ & $S_{60}$ & $S_{100}$ & $\frac{S_{60}}{S_{100}}$ & $\begin{array}{c}L_{\mathrm{FIR}} \\
\left(10^{12} \mathrm{~L}_{\odot}\right)\end{array}$ & $\begin{array}{c}\frac{L_{\mathrm{FIR}}}{M^{\prime}\left(\mathrm{H}_{2}\right)} \\
\frac{\mathrm{L}_{\odot}}{\mathrm{M}_{\odot}}\end{array}$ & $\begin{array}{l}\frac{L_{\mathrm{FIR}}}{L_{\mathrm{CO}}^{\prime}} \\
\frac{\mathrm{L}_{\odot}}{\mathrm{L}_{l}}\end{array}$ \\
\hline $15030+4835$ & 12.5 & 5.8 & $<0.08$ & 0.90 & 1.46 & 0.62 & 1.70 & 29 & 135 \\
\hline Arp 220 & 7.0 & 3.2 & 7.91 & 103.80 & 112.40 & 0.92 & 1.16 & 35 & 164 \\
\hline $16090-0139$ & 12.2 & 5.6 & 0.26 & 3.61 & 4.87 & 0.74 & 2.29 & 40 & 188 \\
\hline $16334+4630$ & 9.5 & 4.4 & $<0.10$ & 1.19 & 2.09 & 0.57 & 1.80 & 41 & 189 \\
\hline NGC 6240 & 7.9 & 3.7 & 3.42 & 22.68 & 27.78 & 0.82 & 0.45 & 12 & 56 \\
\hline $17208-0014$ & 7.1 & 3.2 & 1.66 & 34.14 & 34.90 & 0.98 & 2.14 & 65 & 302 \\
\hline $18368+3549$ & 8.5 & 3.9 & $<0.25$ & 2.23 & 3.84 & 0.58 & 1.20 & 30 & 140 \\
\hline $19297-0406$ & 9.4 & 4.3 & 0.59 & 7.05 & 7.72 & 0.91 & 1.80 & 41 & 191 \\
\hline $19458+0944$ & 12.0 & 5.5 & $<0.28$ & 3.94 & 7.11 & 0.55 & 1.59 & 28 & 132 \\
\hline $20087-0308$ & 16.1 & 7.4 & 0.24 & 4.70 & 6.54 & 0.72 & 1.87 & 25 & 116 \\
\hline $22542+0833$ & 6.0 & 2.8 & $<0.18$ & 1.20 & 1.48 & 0.81 & 1.18 & 42 & 196 \\
\hline $23365+3604$ & 8.5 & 3.9 & 0.81 & 7.09 & 8.36 & 0.85 & 1.01 & 25 & 119 \\
\hline median: & 8 & - & - & - & - & 0.8 & 1.3 & 35 & 160 \\
\hline
\end{tabular}

$L_{\mathrm{FIR}}=3.94 \times 10^{5} r\left(S_{60} / S_{100}\right) \cdot\left(2.58 S_{60}+S_{100}\right) D_{L}^{2}$, with $L_{\mathrm{FIR}}$ in $\mathrm{L}_{\odot}, S$ in $\mathrm{Jy}, D_{L}$ in Mpc, where $D_{L}=c H_{0}^{-1} q_{0}^{-2}\left\{z q_{0}+\left(q_{0}-1\right)\left[\left(2 q_{0} z+1\right)^{0.5}-1\right]\right\} \quad$ (e.g., Weinberg 1972).

We adopt $H_{0}=75 \mathrm{~km} \mathrm{~s}^{-1} \mathrm{Mpc}^{-1}$ and $q_{0}=0.5$.

$\mathrm{L}_{l} \equiv \mathrm{K} \mathrm{km} \mathrm{s}^{-1} \mathrm{pc}^{2}, \quad M^{\prime}\left(\mathrm{H}_{2}\right)=4.6 \cdot L_{\mathrm{CO}}^{\prime}$. 
Table 3. Radii and Dynamical Masses Derived from CO Data

\begin{tabular}{|c|c|c|c|c|c|c|}
\hline $\begin{array}{l}\text { Source } \\
\text { name }\end{array}$ & $\begin{array}{c}\text { bb Temp. } \\
T_{\mathrm{bb}} \\
(\mathrm{K})\end{array}$ & $\begin{array}{c}\text { bb radius } \\
R_{\mathrm{bb}} \\
(\mathrm{pc})\end{array}$ & $\begin{array}{c}\text { CO radius } \\
R_{\mathrm{CO}} \\
(\mathrm{pc})\end{array}$ & $\begin{array}{c}\text { Dyn. Mass } \\
M_{\text {dyn }}\left(<R_{\mathrm{CO}}\right) \\
\left(10^{8} \mathrm{M}_{\odot}\right)\end{array}$ & $\begin{array}{c}\frac{M_{\mathrm{dyn}}}{L_{\mathrm{CO}}^{\prime}} \\
\left(\frac{\mathrm{M}_{\odot}}{\mathrm{L}_{l}}\right)\end{array}$ & $\begin{array}{c}\text { Density } \\
n_{\text {tot }} \\
\left(\mathrm{cm}^{-3}\right)\end{array}$ \\
\hline $00057+4021$ & 72 & 81 & 219 & 62 & 1.6 & 2850 \\
\hline $00188-0856$ & 62 & 238 & 297 & 105 & 1.6 & 1932 \\
\hline $00262+4251$ & 88 & 98 & 314 & 66 & 1.1 & 1023 \\
\hline $\mathrm{I} \mathrm{Zw} 1$ & 63 & 101 & 248 & 97 & 1.9 & 3060 \\
\hline Mrk 1014 & 80 & 174 & 418 & 87 & 1.0 & 576 \\
\hline $02483+4302$ & 50 & 197 & 273 & 57 & 2.0 & 1352 \\
\hline $03158+4227$ & 75 & 220 & 405 & 85 & 1.2 & 613 \\
\hline $03521+0028$ & 60 & 319 & 575 & 120 & 1.3 & 304 \\
\hline $04232+1436$ & 62 & 172 & 340 & 126 & 1.4 & 1544 \\
\hline VII Zw 31 & 50 & 244 & 616 & 129 & 1.1 & 265 \\
\hline $07598+6508$ & 76 & 153 & 377 & 99 & 0.9 & 893 \\
\hline $08030+5243$ & 56 & 208 & 345 & 141 & 1.6 & 1653 \\
\hline $08572+3915$ & 119 & 53 & 113 & 24 & 1.8 & 7862 \\
\hline $09320+6134$ & 49 & 266 & 295 & 84 & 1.8 & 1570 \\
\hline $10035+4852$ & 58 & 186 & 395 & 82 & 1.2 & 646 \\
\hline $10190+1322$ & 52 & 243 & 360 & 127 & 1.5 & 1316 \\
\hline $10495+4424$ & 55 & 265 & 379 & 96 & 1.2 & 846 \\
\hline $10565+2448$ & 59 & 188 & 317 & 66 & 1.2 & 1000 \\
\hline $11506+1331$ & 63 & 229 & 361 & 75 & 1.0 & 772 \\
\hline Mrk 231 & 73 & 198 & 378 & 79 & 1.0 & 702 \\
\hline 13106-0922 & 61 & 281 & 496 & 104 & 1.1 & 410 \\
\hline Arp 193 & 50 & 170 & 244 & 95 & 2.5 & 3156 \\
\hline Mrk 273 & 70 & 157 & 280 & 59 & 1.2 & 1284 \\
\hline $13442+2321$ & 61 & 221 & 441 & 92 & 1.8 & 518 \\
\hline $14070+0525$ & 72 & 284 & 394 & 82 & 0.9 & 648 \\
\hline
\end{tabular}


Table 3-Continued

\begin{tabular}{|c|c|c|c|c|c|c|}
\hline $\begin{array}{l}\text { Source } \\
\text { name }\end{array}$ & $\begin{array}{c}\text { bb Temp. } \\
T_{\mathrm{bb}} \\
(\mathrm{K})\end{array}$ & $\begin{array}{c}\text { bb radius } \\
\qquad R_{\mathrm{bb}} \\
(\mathrm{pc})\end{array}$ & $\begin{array}{c}\text { CO radius } \\
\qquad R_{\mathrm{CO}} \\
(\mathrm{pc})\end{array}$ & $\begin{array}{c}\text { Dyn. Mass } \\
M_{\text {dyn }}\left(<R_{\mathrm{CO}}\right) \\
\left(10^{8} \mathrm{M}_{\odot}\right)\end{array}$ & $\begin{array}{l}\frac{M_{\mathrm{dyn}}}{L_{\mathrm{CO}}^{\prime}} \\
\left(\frac{\mathrm{M}_{\odot}}{\mathrm{L}_{l}}\right)\end{array}$ & $\begin{array}{c}\text { Density } \\
n_{\text {tot }} \\
\left(\mathrm{cm}^{-3}\right)\end{array}$ \\
\hline $15030+4835$ & 59 & 281 & 499 & 104 & 0.8 & 404 \\
\hline Arp 220 & 64 & 197 & 270 & 144 & 2.1 & 3549 \\
\hline $16090-0139$ & 61 & 304 & 459 & 96 & 0.8 & 477 \\
\hline $16334+4630$ & 56 & 327 & 413 & 98 & 1.0 & 673 \\
\hline NGC 6240 & 59 & 146 & 341 & 108 & 1.4 & 1320 \\
\hline $17208-0014$ & 69 & 235 & 301 & 91 & 1.3 & 1602 \\
\hline $18368+3549$ & 53 & 299 & 396 & 100 & 1.2 & 775 \\
\hline $19297-0406$ & 68 & 222 & 385 & 80 & 0.9 & 680 \\
\hline $19458+0944$ & 51 & 373 & 465 & 132 & 1.1 & 633 \\
\hline $20087-0308$ & 59 & 297 & 466 & 173 & 1.1 & 824 \\
\hline $22542+0833$ & 67 & 182 & 436 & 91 & 1.5 & 529 \\
\hline $23365+3604$ & 63 & 191 & 372 & 83 & 1.0 & 777 \\
\hline
\end{tabular}

$$
\begin{aligned}
& T_{\mathrm{bb}} \approx-(1+z)\left(\frac{82}{\ln \left(0.3 \cdot S_{60} / S_{100}\right)}-0.5\right) ; \quad R_{\mathrm{bb}}=\left(L_{\mathrm{FIR}} /\left(4 \pi \sigma T_{\mathrm{bb}}^{4}\right)^{0.5}\right. \\
& R_{\mathrm{CO}}(\min )=\left(L_{\mathrm{CO}}^{\prime} /\left(\pi T_{\mathrm{bb}} \Delta V\right)^{0.5} ; \quad\right. \\
& M_{\mathrm{dyn}}\left(<R_{\mathrm{CO}}\right)=232 \cdot R_{\mathrm{CO}} \cdot[\operatorname{Max}(300, \Delta V)]^{2}
\end{aligned}
$$


Table 4. Mass Estimates for Ultraluminous Galaxies

\begin{tabular}{|c|c|c|c|c|}
\hline $\begin{array}{l}\text { Source } \\
\text { name }\end{array}$ & $\begin{array}{c}M_{\mathrm{dyn}}\left(<R_{\mathrm{CO}}\right) \\
\left(10^{8} \mathrm{M}_{\odot}\right)\end{array}$ & $\begin{array}{c}M^{\prime}\left(\mathrm{H}_{2}\right) \\
\left(10^{8} \mathrm{M}_{\odot}\right)\end{array}$ & $\begin{array}{c}M_{\text {thin }} \\
\left(10^{8} \mathrm{M}_{\odot}\right)\end{array}$ & $\begin{array}{l}100 M_{\text {dust }} \\
\left(10^{8} \mathrm{M}_{\odot}\right)\end{array}$ \\
\hline $00057+4021$ & 62 & 175 & 19 & 15 \\
\hline $00188-0856$ & 105 & 309 & 29 & 130 \\
\hline $00262+4251$ & 65 & 286 & 38 & 28 \\
\hline I Zw 1 & 97 & 230 & 22 & 22 \\
\hline Mrk 1014 & 87 & 402 & 48 & 76 \\
\hline $02483+4302$ & 57 & 133 & 10 & 82 \\
\hline $03158+4227$ & 85 & 322 & 37 & 115 \\
\hline $03521+0028$ & 120 & 427 & 38 & 207 \\
\hline $04232+1436$ & 126 & 413 & 38 & 61 \\
\hline VII Zw 31 & 129 & 544 & 41 & 111 \\
\hline $07598+6508$ & 99 & 523 & 60 & 57 \\
\hline $08030+5243$ & 141 & 406 & 34 & 44 \\
\hline $08572+3915$ & 24 & 60 & 11 & 46 \\
\hline $09320+6134$ & 84 & 214 & 16 & 26 \\
\hline $10035+4852$ & 82 & 324 & 28 & 119 \\
\hline $10190+1322$ & 127 & 378 & 30 & 88 \\
\hline $10495+4424$ & 96 & 376 & 31 & 168 \\
\hline $10565+2448$ & 66 & 258 & 23 & 82 \\
\hline $11506+1331$ & 75 & 345 & 33 & 148 \\
\hline Mrk 231 & 79 & 347 & 38 & 92 \\
\hline $13106-0922$ & 103 & 431 & 40 & 160 \\
\hline Arp 193 & 95 & 176 & 13 & 57 \\
\hline Mrk 273 & 59 & 230 & 25 & 56 \\
\hline $13442+2321$ & 92 & 239 & 22 & 106 \\
\hline $14070+0525$ & 82 & 435 & 47 & 188 \\
\hline
\end{tabular}


Table 4-Continued

\begin{tabular}{|c|c|c|c|c|}
\hline $\begin{array}{l}\text { Source } \\
\text { name }\end{array}$ & $\begin{array}{l}M_{\text {dyn }}\left(<R_{\mathrm{CO}}\right) \\
\quad\left(10^{8} \mathrm{M}_{\odot}\right)\end{array}$ & $\begin{array}{c}M^{\prime}\left(\mathrm{H}_{2}\right) \\
\left(10^{8} \mathrm{M}_{\odot}\right)\end{array}$ & $\begin{array}{c}M_{\text {thin }} \\
\left(10^{8} \mathrm{M}_{\odot}\right)\end{array}$ & $\begin{array}{l}100 M_{\text {dust }} \\
\left(10^{8} \mathrm{M}_{\odot}\right)\end{array}$ \\
\hline $15030+4835$ & 104 & 575 & 51 & 164 \\
\hline Arp 220 & 144 & 323 & 31 & 84 \\
\hline $16090-0139$ & 96 & 560 & 52 & 195 \\
\hline $16334+4630$ & 98 & 438 & 37 & 208 \\
\hline NGC 6240 & 108 & 366 & 33 & 44 \\
\hline $17208-0014$ & 91 & 325 & 34 & 123 \\
\hline $18368+3549$ & 100 & 393 & 31 & 171 \\
\hline 19297-0406 & 80 & 434 & 44 & 107 \\
\hline $19458+0944$ & 132 & 552 & 42 & 261 \\
\hline $20087-0308$ & 173 & 740 & 66 & 184 \\
\hline $22542+0833$ & 91 & 277 & 28 & 101 \\
\hline $23365+3604$ & 83 & 390 & 37 & 77 \\
\hline
\end{tabular}


Table 5. Optical / Near-IR Morphology of Ultraluminous Galaxies

\begin{tabular}{|c|c|c|c|c|c|c|}
\hline $\begin{array}{l}\text { Source } \\
\text { name }\end{array}$ & $\begin{array}{l}\text { Redshift D } \\
\qquad z z \\
\left(\mathrm{~km} \mathrm{~s}^{-1}\right)\end{array}$ & $\begin{array}{l}\text { istance } \\
D_{A} \\
\mathrm{Mpc})\end{array}$ & Morphology & $\begin{array}{c}\text { Separation } \\
\theta \\
(\operatorname{arcsec})\end{array}$ & $\begin{array}{c}D_{A} \cdot \theta \\
(\mathrm{kpc})\end{array}$ & Ref. \\
\hline $00057+4021$ & 13390 & 165 & Double? disturbed & $2.5 ?$ & 2 & AHM87 \\
\hline $00188-0856$ & 38530 & 415 & Merger + companion & 7 & 14 & M96 \\
\hline $00262+4251$ & 29153 & 330 & Single & $<0.8$ & $<1.3$ & M96 \\
\hline I Zw 1 & 18330 & 220 & Single (quasar) & - & - & \\
\hline Mrk 1014 & 48947 & 501 & Single (quasar) & - & - & \\
\hline $02483+4302$ & 15419 & 188 & Merger & 3.8 & 3.5 & KDBS \\
\hline $03158+4227$ & 40296 & 431 & Single & $<0.8$ & $<1.6$ & M96 \\
\hline $03521+0028$ & 45530 & 474 & Double & 1.6 & 3.6 & M96 \\
\hline $04232+1436$ & 23855 & 278 & Double, merger & 4.6 & 5 & \\
\hline VII Zw 31 & 16260 & 196 & Single (starburst) & - & - & \\
\hline $07598+6508$ & 44621 & 466 & Single (quasar) & - & - & HB89, S92 \\
\hline $08030+5243$ & 25031 & 290 & Single & $<0.8$ & $<1.1$ & M96 \\
\hline $08572+3915$ & 17450 & 211 & Double & 6 & 5.5 & S92 \\
\hline $09320+6134$ & 11785 & 147 & Single; tails & $<1.0$ & $<0.7$ & S92, M96 \\
\hline $10035+4852$ & 19427 & 232 & - & - & - & \\
\hline $10190+1322$ & 22953 & 269 & Double & 5 & 6.6 & Fig. 7 \\
\hline $10495+4424$ & 27674 & 315 & Single, distorted & $<0.7$ & $<1.1$ & Fig. 7, M96 \\
\hline $10565+2448$ & 12923 & 160 & Double, Multiple & 8,26 & 6,20 & Fig. 7, M96 \\
\hline $11506+1331$ & 38158 & 412 & Double, Single & $<0.5$ & $<1.1$ & Fig. 7, M96 \\
\hline Mrk 231 & 12650 & 157 & Double nucl.?, tail & 3.5 & 2.8 & A94 \\
\hline $13106-0922$ & 52290 & 526 & Double & 2 & 5 & Fig. 7 \\
\hline Arp 193 & 7000 & 90 & Single +2 tails & $>1 ?$ & $>4 \quad ?$ & S92 \\
\hline Mrk 273 & 11324 & 142 & Double in NIR, 2 tails & 0.9 & 0.6 & M93 \\
\hline $13442+2321$ & 42620 & 450 & Merger? & - & - & \\
\hline $14070+0525$ & 79621 & 702 & Single & - & - & Fig. 7 \\
\hline
\end{tabular}


Table 5-Continued

\begin{tabular}{|c|c|c|c|c|c|c|}
\hline $\begin{array}{l}\text { Source } \\
\text { name }\end{array}$ & $\begin{array}{c}\text { Redshift D } \\
\qquad z \\
\left(\mathrm{~km} \mathrm{~s}^{-1}\right)\end{array}$ & $\begin{array}{l}\text { istance } \\
D_{A} \\
\mathrm{Mpc})\end{array}$ & Morphology & $\begin{array}{c}\text { Separation } \\
\theta \\
(\operatorname{arcsec})\end{array}$ & $\begin{array}{l}D_{A} \cdot \theta \\
(\mathrm{kpc})\end{array}$ & Ref. \\
\hline $15030+4835$ & 64900 & 613 & Double & 3.5 & 11 & Fig. 7 \\
\hline Arp 220 & 5450 & 70 & Merger, double nuc.? & 1 & 0.3 & G90, M93, S94 \\
\hline $16090-0139$ & 40044 & 429 & Single, distorted & $<1.0$ & $<2.2$ & Fig. 7, M96 \\
\hline $16334+4630$ & 57250 & 562 & Double & 4.4 & 12.6 & Fig. 7 \\
\hline NGC 6240 & 7298 & 93 & Double nucl. & - & - & AHM90; L94 \\
\hline $17208-0014$ & 12837 & 159 & Double + tails, Single & $<0.8$ & $<0.6$ & Fig. 7, M96 \\
\hline $18368+3549$ & 34850 & 383 & Single & $<0.8$ & $<1.5$ & M96 \\
\hline $19297-0406$ & 25700 & 297 & Single & $<1.9$ & $<2.7$ & Fig. 7, M96 \\
\hline $19458+0944$ & 29980 & 338 & Double?, Single & $<0.8$ & $<1.4$ & M96 \\
\hline $20087-0308$ & 31688 & 354 & Single, tails & $<1.0$ & $<1.7$ & MM90, M96 \\
\hline $22542+0833$ & 49750 & 487 & Double & - & - & \\
\hline $23365+3604$ & 19330 & 231 & Single, tails & $<0.9$ & $<1.1$ & Fig. 7, M96 \\
\hline
\end{tabular}

$D_{A}=D_{L} /(1+z)^{2} ;$ for $D_{L}$, see footnote to Table 2 .

A87, A90, A94: Armus et al. (1987; 1990; 1994); G90: Graham et al. (1990);

KDBS: Kollatschny et al. (1991); L94: Lester \& Gaffney (1994);

M93: Majewski et al. (1993); M96: Murphy et al. (1996);

MM90: Melnick \& Mirabel (1990); S92: Sanders (1992);

S94: Shaya et al. (1994). 
Fig. 1.- Distribution of redshifts in our sample of ultraluminous galaxies.

Fig. 2.- $\mathrm{CO}(1-0)$ spectra of galaxies in our sample. The vertical scale is main-beam brightness temperature, in $\mathrm{mK}$. Source names and redshifts are indicated in the boxes. For each source, the zero of the (Doppler) velocity scale is relative to the redshifts listed in Table 1. The spectra are in order of decreasing redshift.

Fig. 3.- FIR luminosity vs. $\mathrm{CO}(1-0)$ luminosity (lower scale) and molecular gas mass (top scale). Solid circles indicate galaxies in our sample. Normal and weakly interacting spirals are scattered about the solid line. Open circles show locations in this diagram for some well-known galaxies.

Fig. 4. - Integrated $\mathrm{CO}(1-0)$ line intensity, $I_{\mathrm{CO}}$, in $\mathrm{K}_{\mathrm{mb}} \mathrm{km} \mathrm{s}^{-1}$, vs. IRAS $100 \mu \mathrm{m}$ flux density, in Jy, for the ultraluminous galaxies in our sample. The solid line corresponds to the relation $I_{\mathrm{CO}}=1.0 \times S_{100}$.

Fig. 5.- The distant-independent quantity $L_{\mathrm{FIR}} / L_{\mathrm{CO}}$ vs. the far IR black body dust temperature derived from the IRAS flux ratio $S_{60} / S_{100}$. The line indicates the upper limit for the luminosity ratio for the black body model in eq.(11), with $f_{V} \Delta V=300 \mathrm{~km} \mathrm{~s}^{-1}$. Solid circles: galaxies in our sample with $L_{\mathrm{FIR}}>10^{12} \mathrm{~L}_{\odot}$. Open circles: galaxies with $L_{\mathrm{FIR}}<10^{12} \mathrm{~L}_{\odot}$.

Fig. 6.- Distribution of observed CO full widths to half-maximum, $\Delta V$, for the galaxies in our sample.

Fig. 7.- R-band CCD images of galaxies between RA $10 \mathrm{~h}$ and $23 \mathrm{~h}$ in the sample. The images are in order of decreasing redshift. North is at the top, east is to the left. Brightness contours are on an arbitrary linear scale. Next to each image is source name, redshift and $\mathrm{CO}(1-0)$ spectrum, with units as in Fig. 1. The images were taken at the Univ. of Hawaii 88-in. telescope by Sanders \& Kim (1993, priv. communication). 


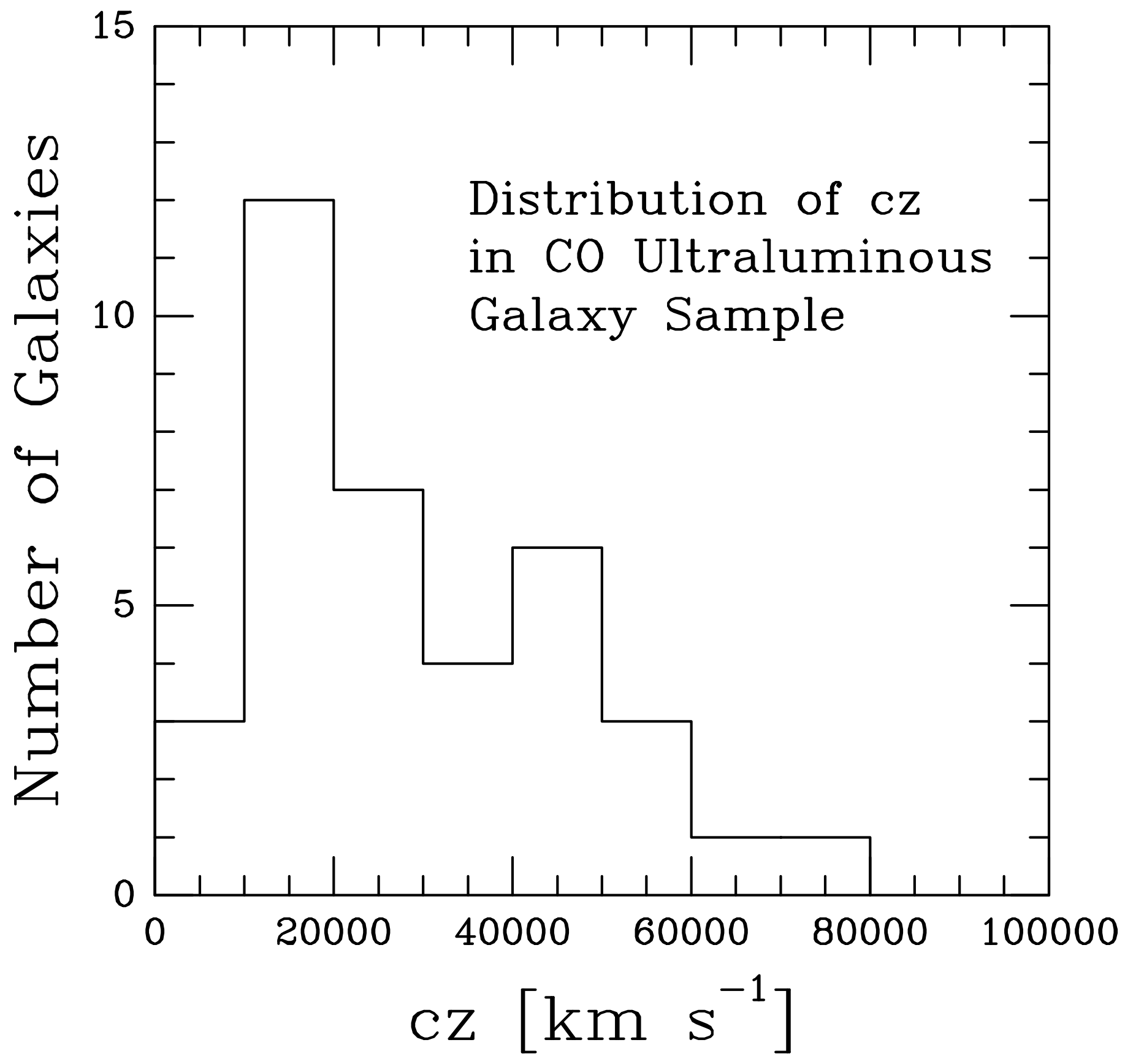



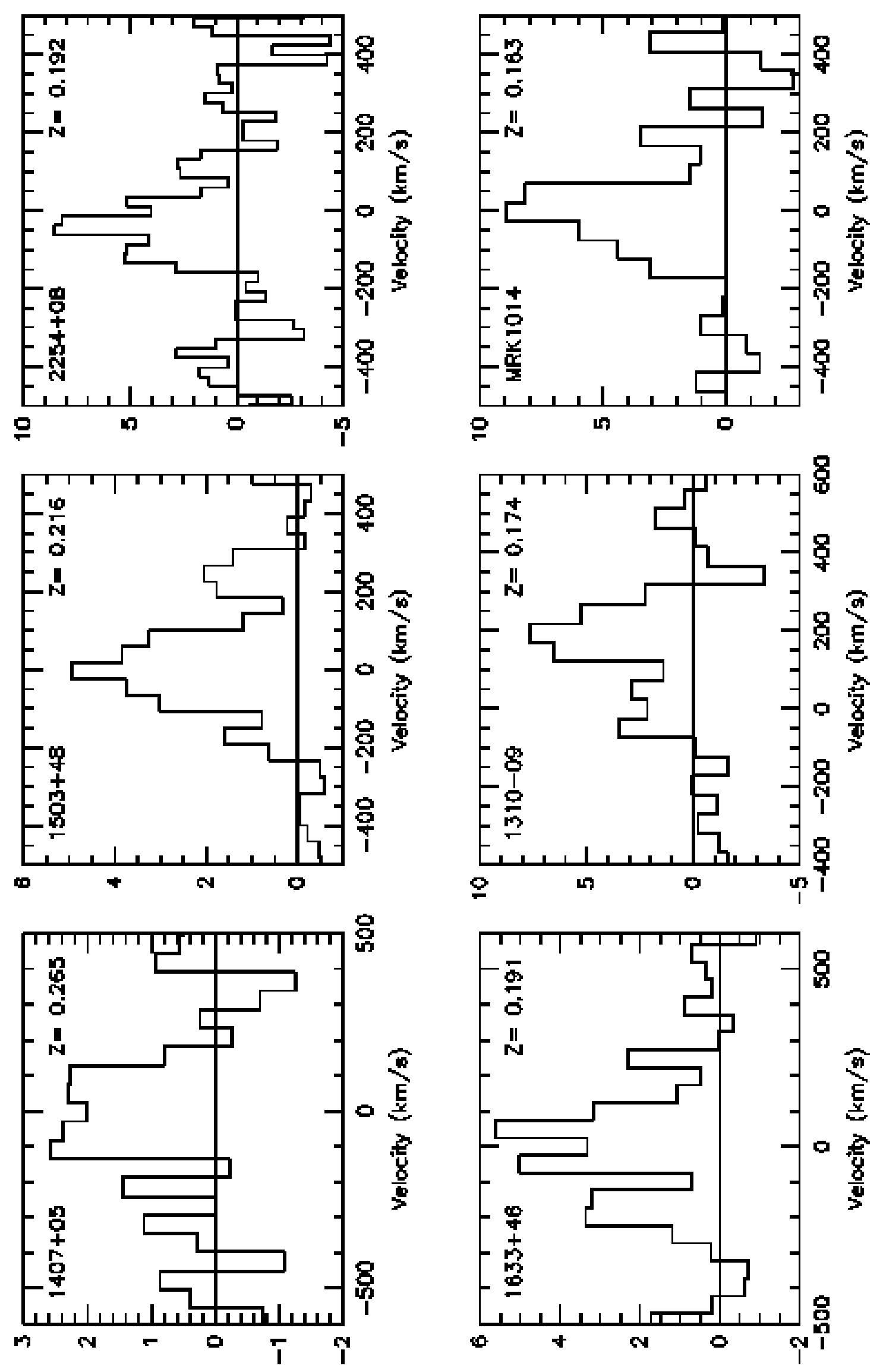

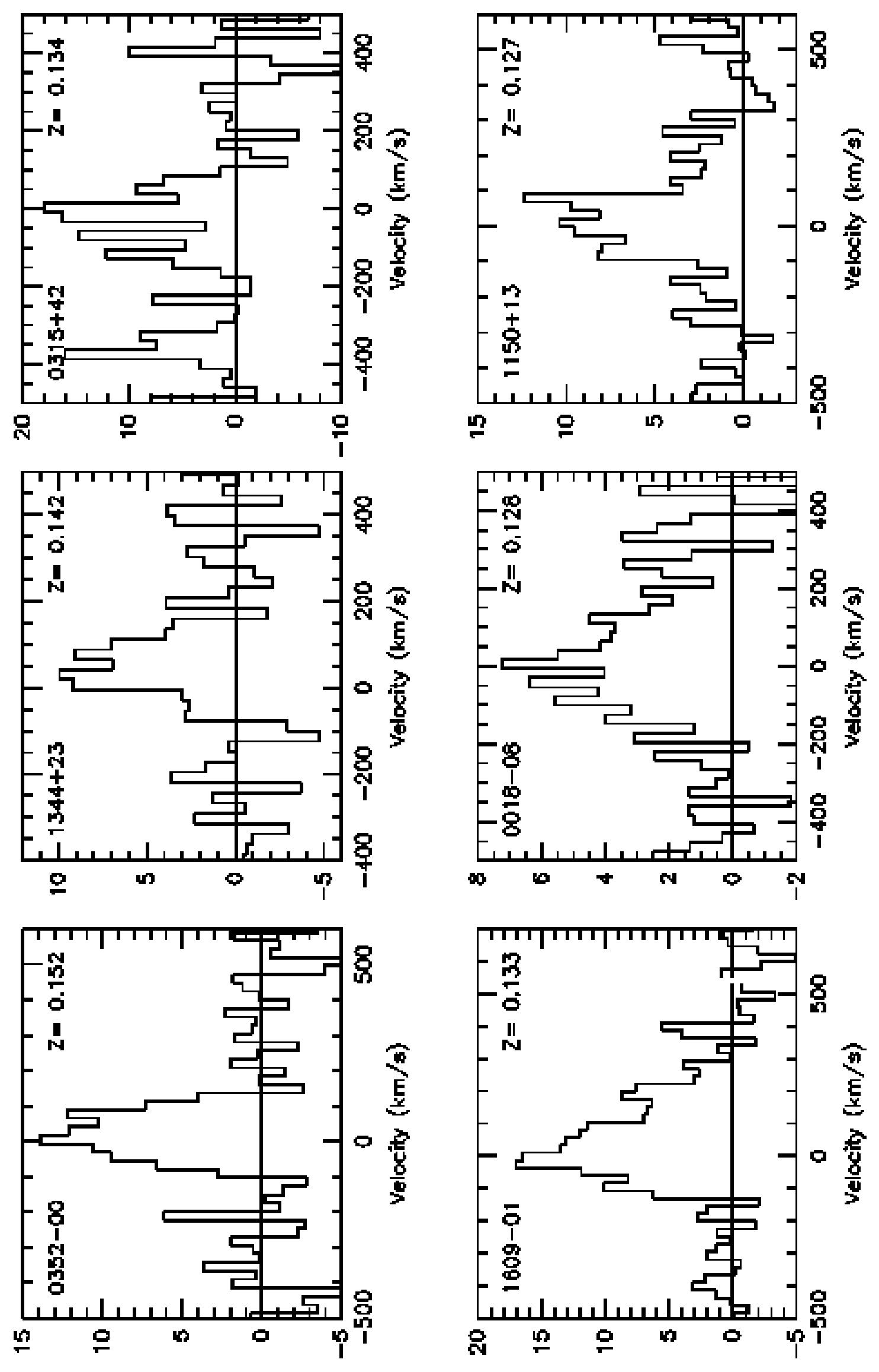

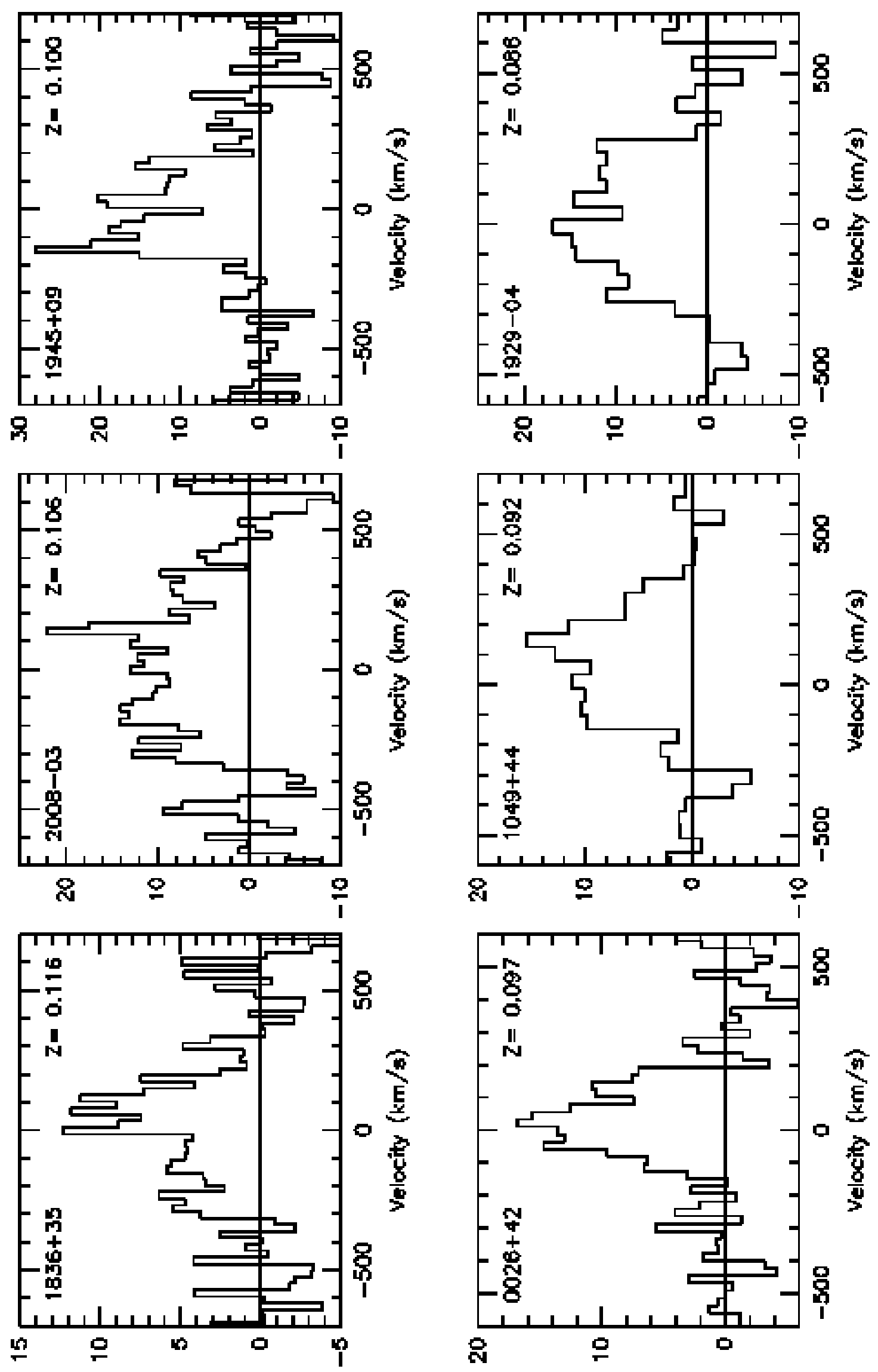

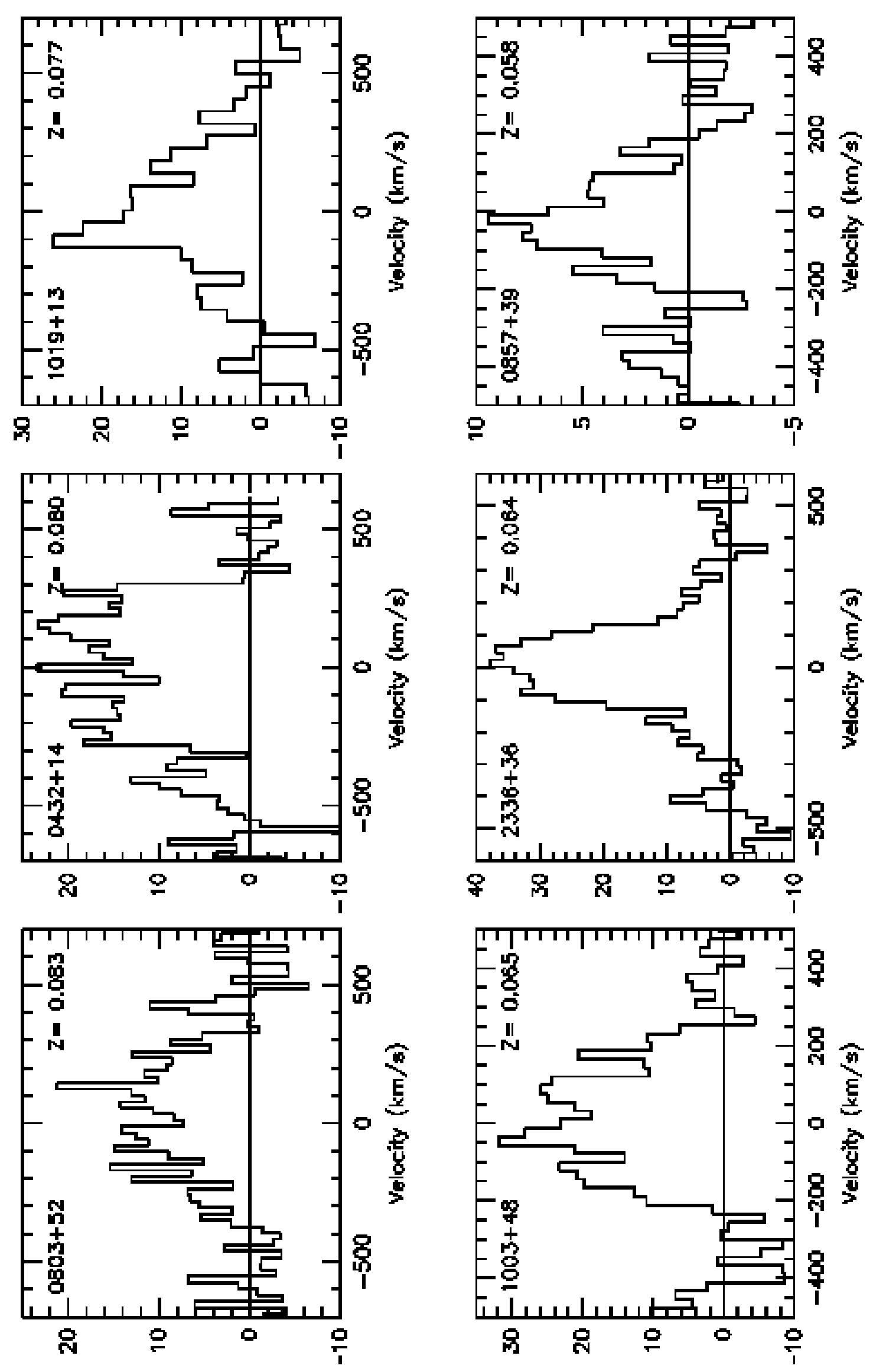

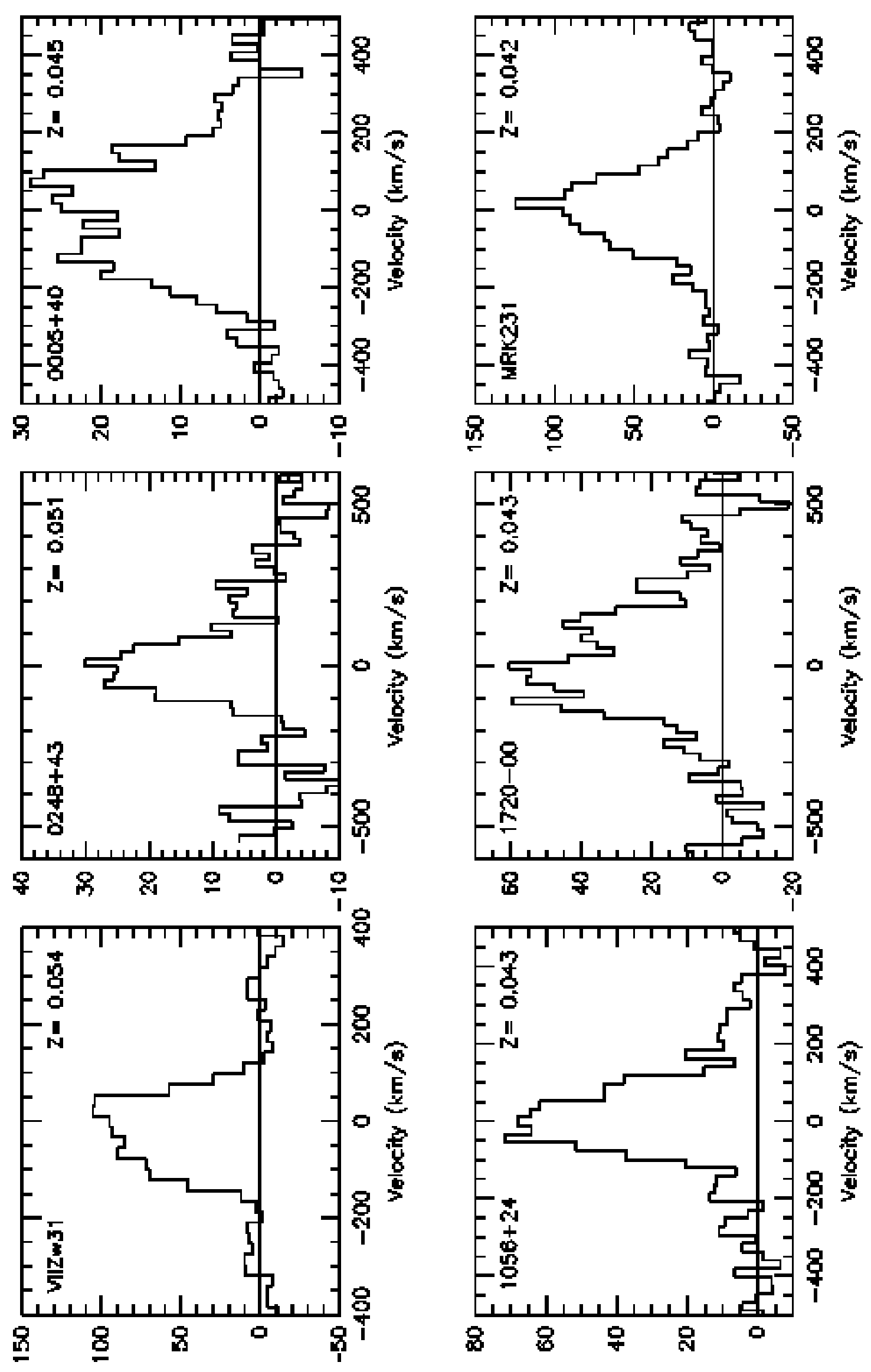

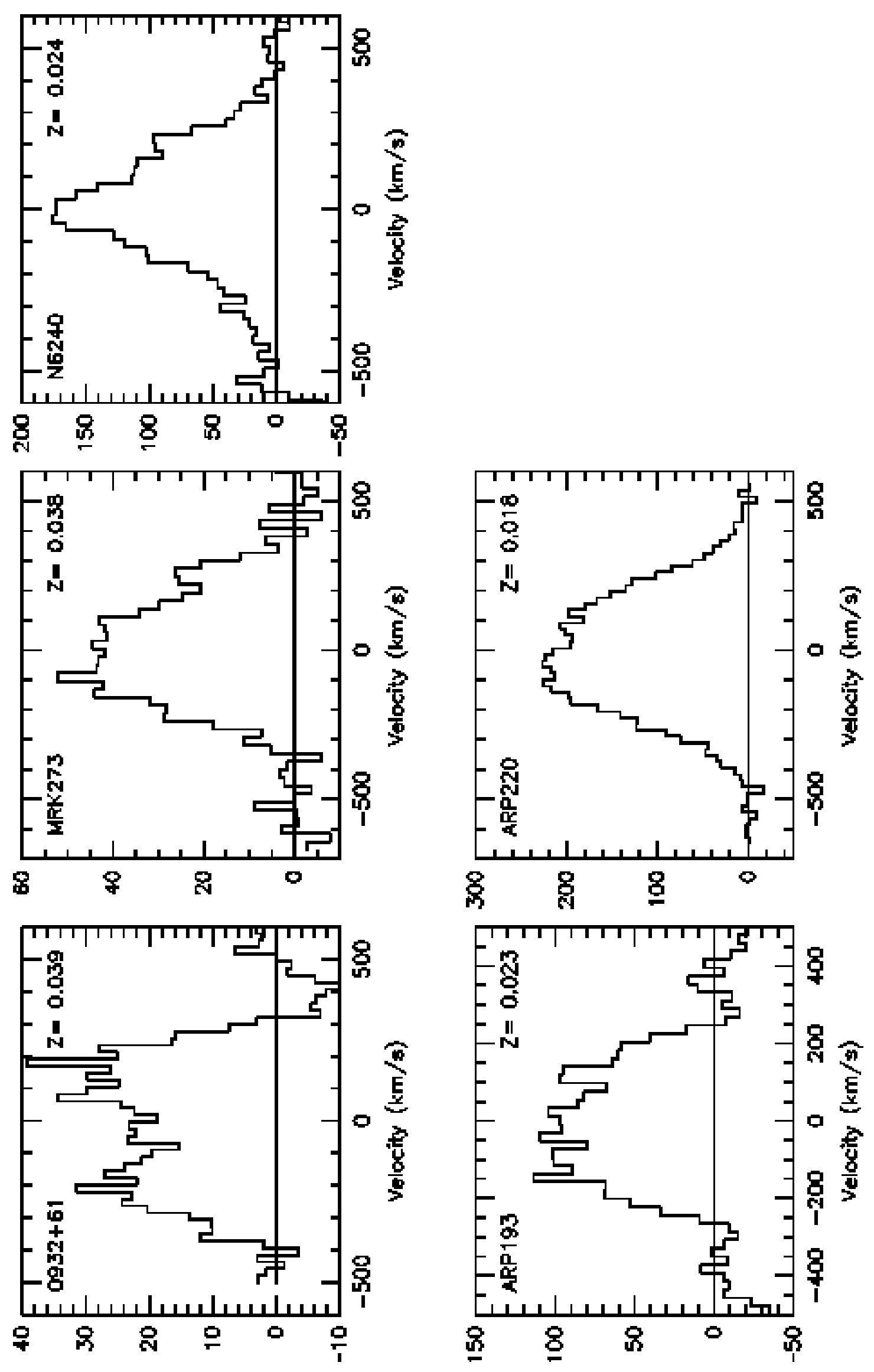


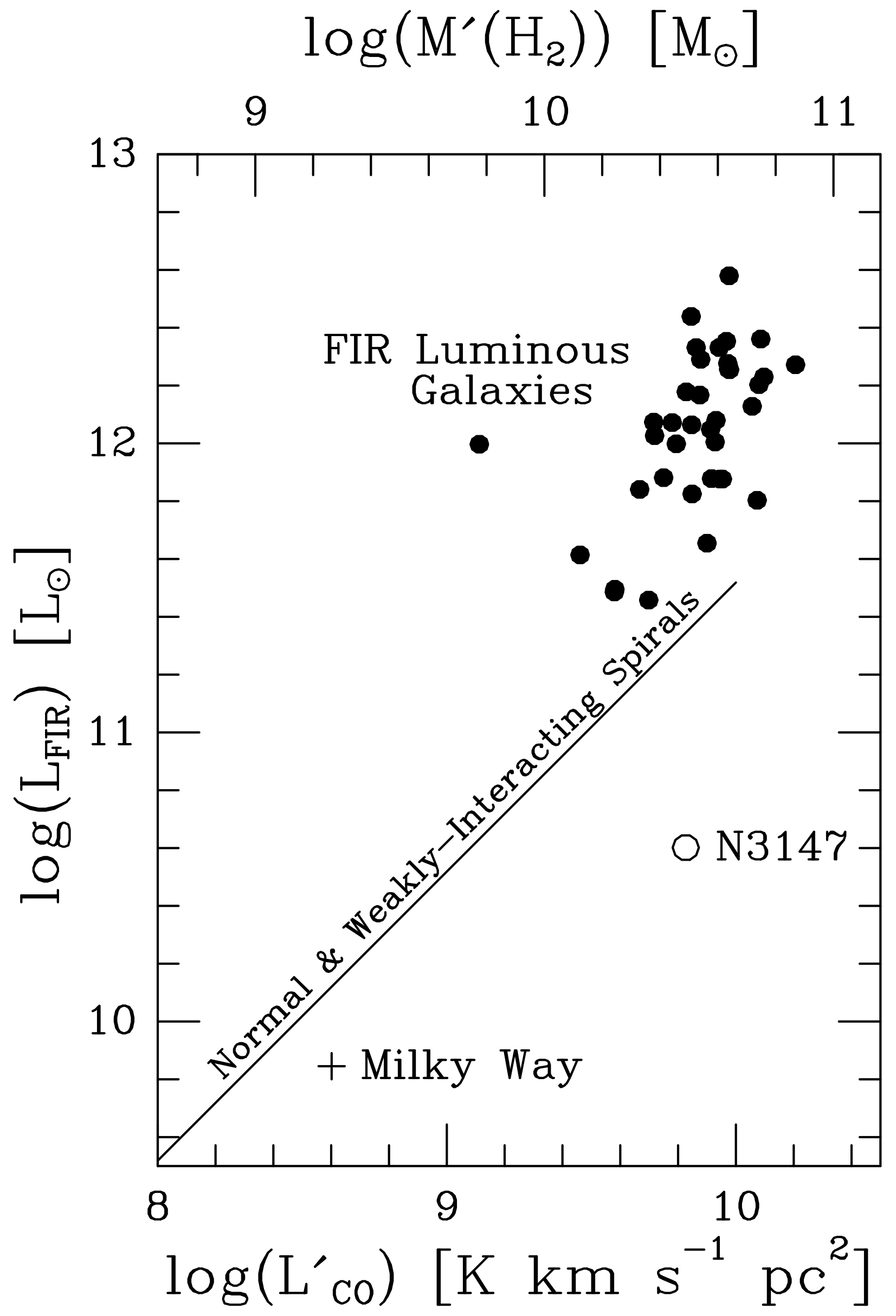




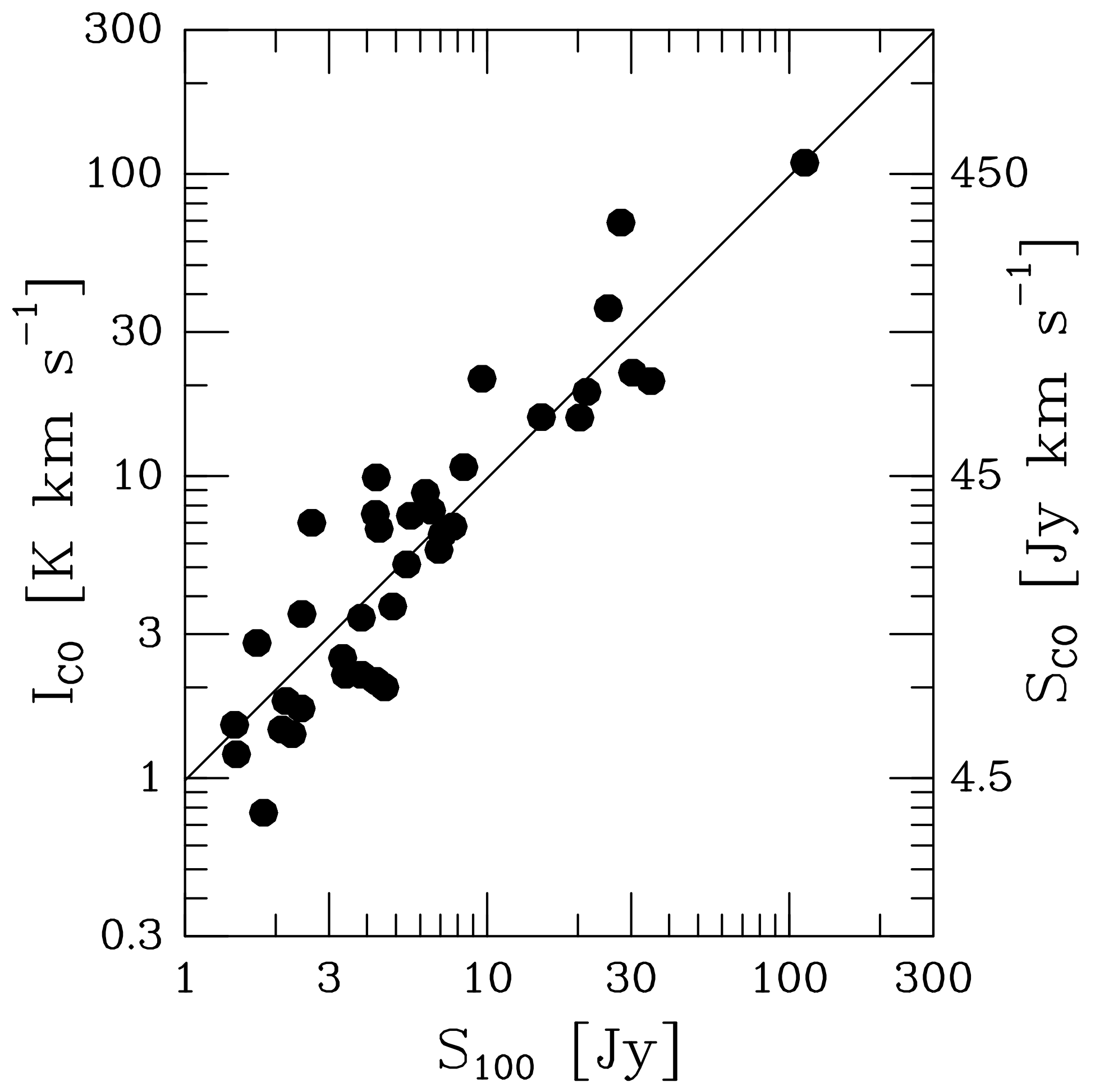




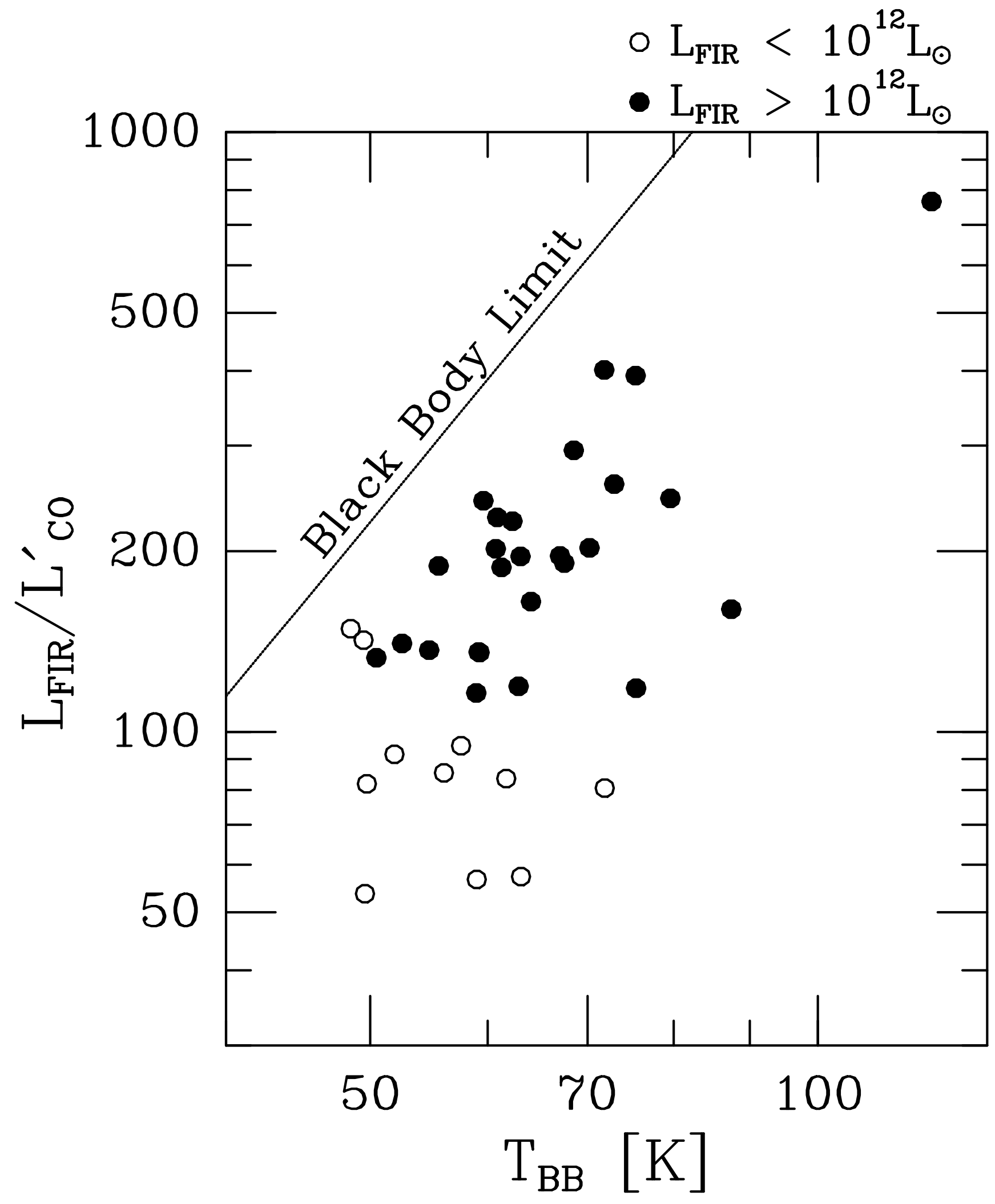




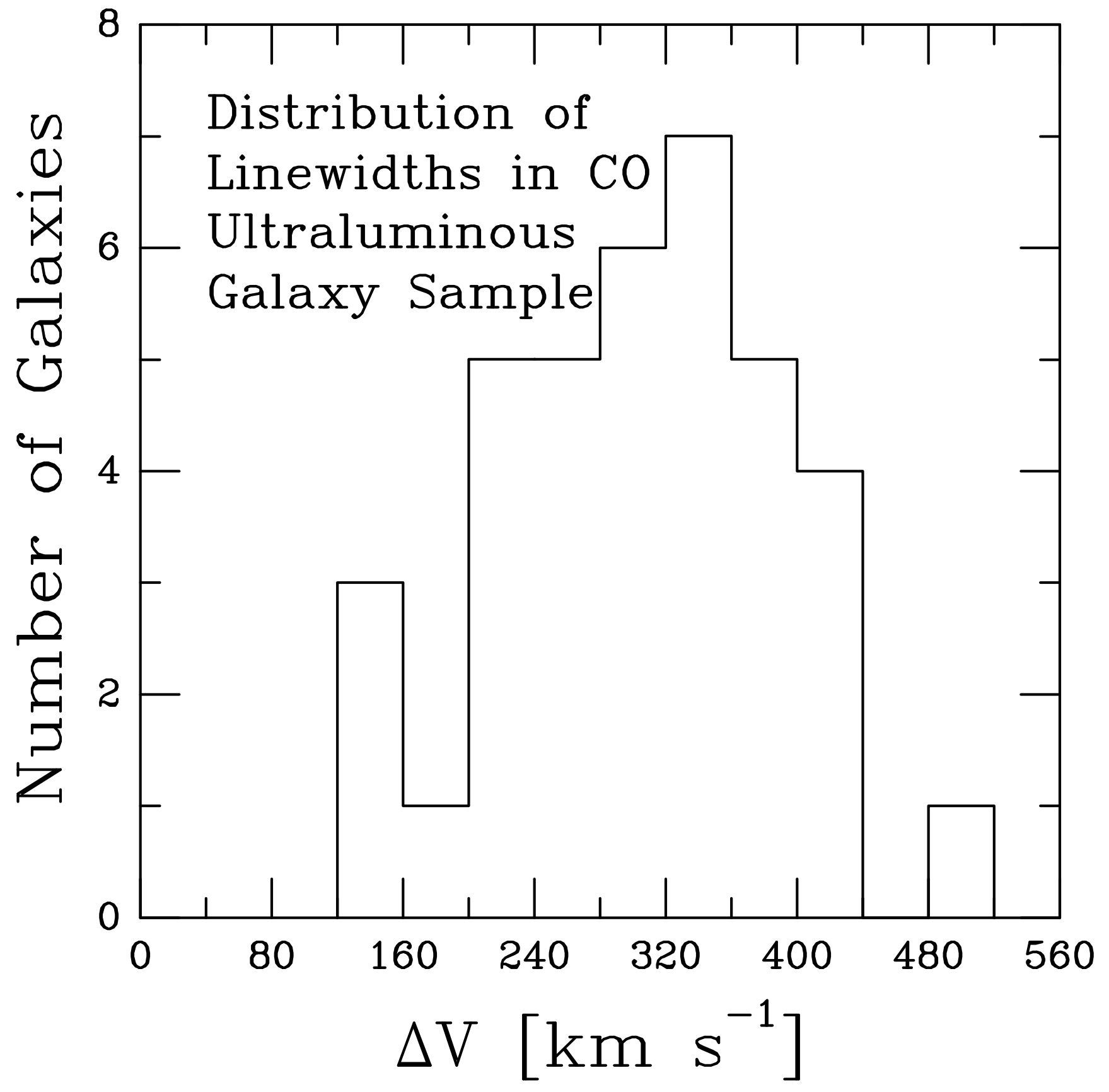



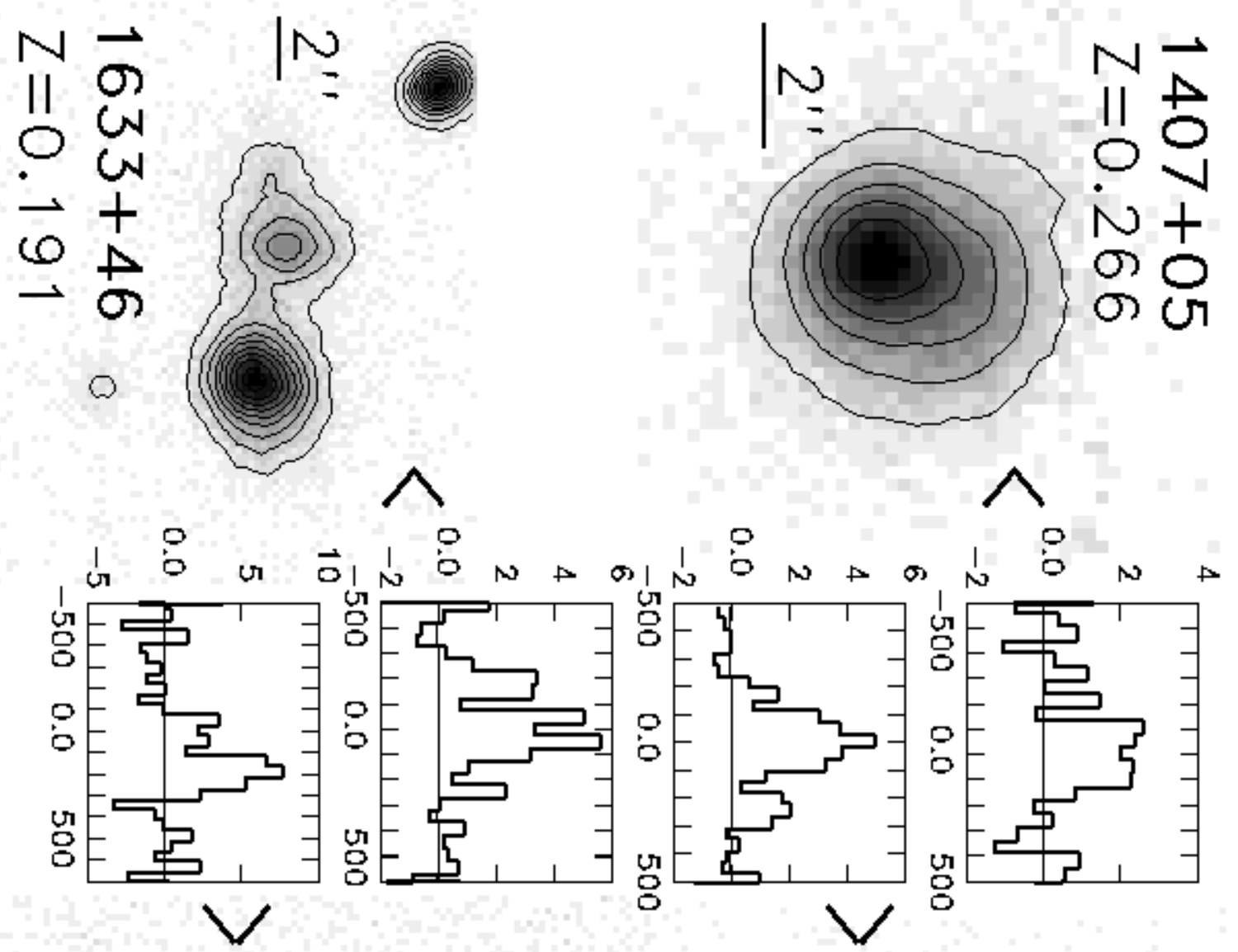

๙

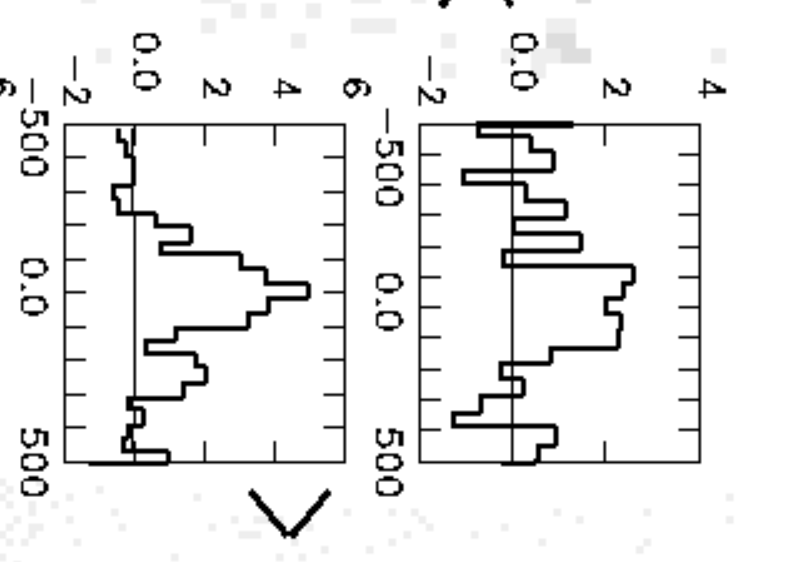

미늠

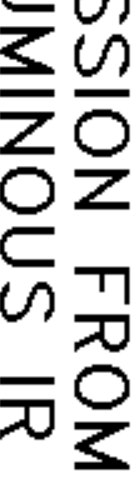

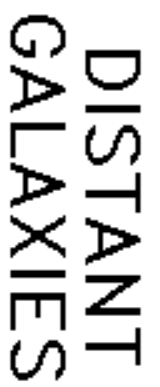
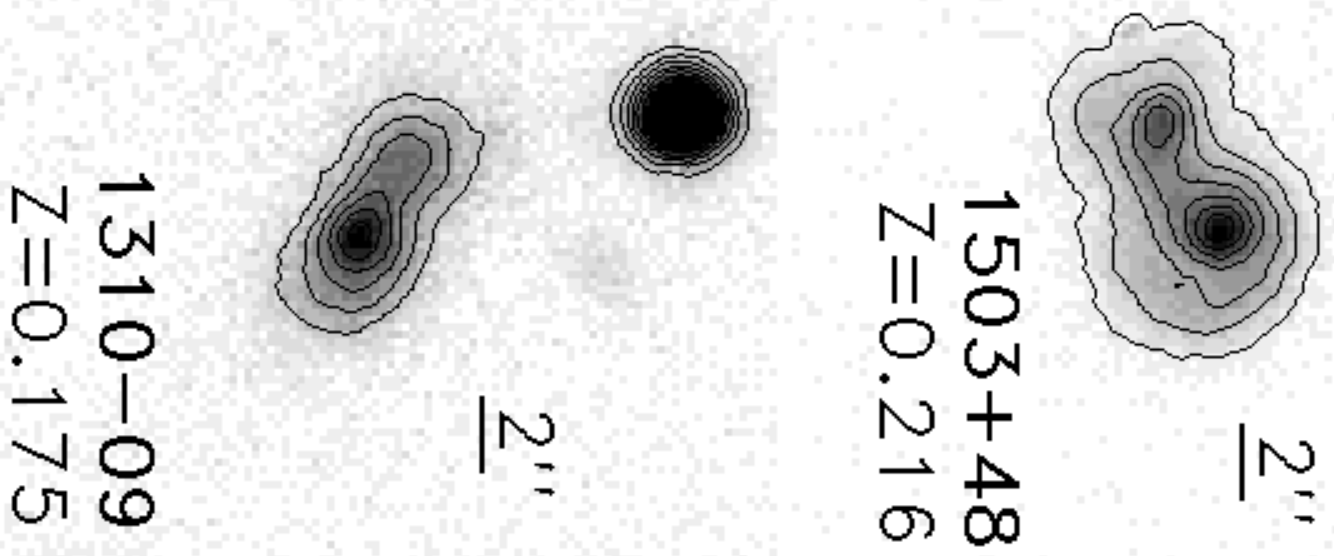


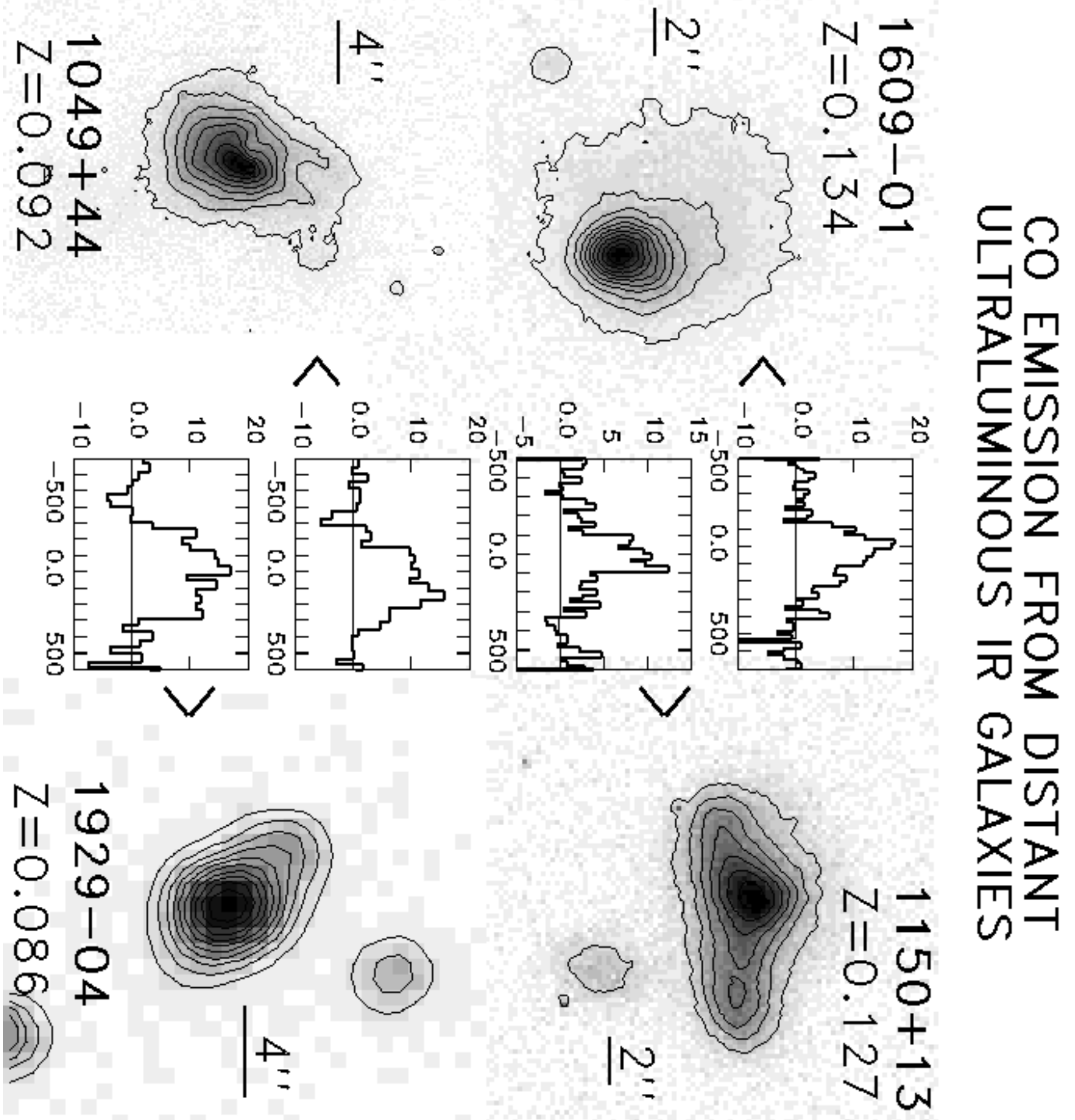




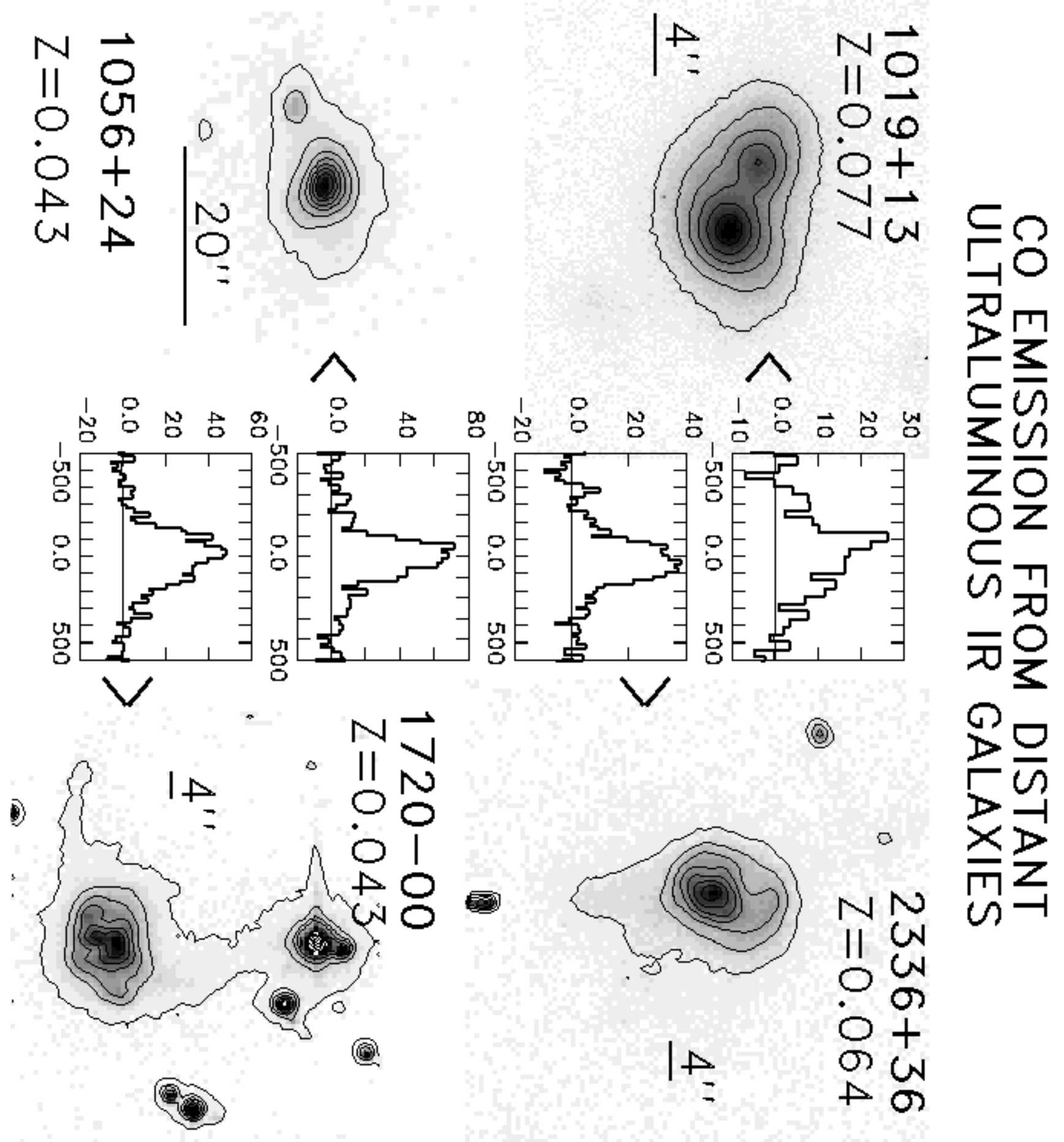

